\title{
Oscillation Modes in Symmetrical Wireless-Locked Systems
}

\author{
Mabel Pontón, Member, IEEE, Almudena Suárez, Fellow, IEEE
}

\begin{abstract}
Time synchronization of multiple elements of a wireless network can be achieved through the wireless coupling of their oscillator circuits. Most previous works on wireless locking of oscillators analyze the system in an idealized manner, representing the oscillator elements with phase models and describing the propagation effects with constant scalar coefficients and time delays. Here a realistic analysis of the wireless system is presented, which relies on the extraction of the oscillator models from harmonic-balance simulations and takes into account the antenna gains and propagation effects. The most usual network configurations, corresponding to ring, fully connected and star topologies, are investigated in detail. In symmetric conditions, the oscillation modes are detected through an eigenvalue/eigenvector calculation of an equivalent coupling matrix. For each particular mode, the system is analyzed in two manners: by means of an analytical formulation, able to provide all the coexistent solutions, and through a circuit-level harmonicbalance simulation of an equivalent system with a reduced number of oscillator elements. The stability properties are determined by means of a perturbation system of general application to any coupled structure. A specific formulation is also derived to predict the impact of discrepancies between the oscillator elements. All the results have been validated with independent circuit-level simulations and measurements.
\end{abstract}

Index Terms-Wireless locking, oscillator, stability, phase noise.

\section{INTRODUCTION}

$\mathrm{T}$ IME synchronization between different devices is a usual requirement in engineering systems, such as computer networks, measurement systems, sensor networks and other [1]-[10]. For instance, the efficient data transmission in a multiple-input multiple output (MIMO) antenna systems, requires phase synchronization between the antennas, which is still a significant challenge [2]. In the case of sensor networks, a common time scale between the sensor nodes [10] enables applications such as cooperative transmissions, data-fusion of time-sensitive measurements or moving object tracking. The synchronization can be achieved through the broadcast of a

Manuscript received July 1, 2017. This work was supported by the Spanish Ministry of Economy and Competitiveness under the research project TEC2014-60283-C3-1-R, the European Regional Development Fund (ERDF/FEDER) and Juan de la Cierva Research Program IJCI-2014-19141 and by the Parliament of Cantabria under the project Cantabria Explora 12.JP02.64069. This paper is an expanded version from the 2017 International Microwave Symposium, Honolulu, HI, 4-9 June 2017.

M. Pontón and A. Suárez are with the Departamento de Ingeniería de Comunicaciones, Universidad de Cantabria, Santander, 39005, Spain (e-mail: mabel.ponton@unican.es; almudena.suarez@unican.es). beacon timing signal from a central node [1]. However, in fully distributed scenarios, this broadcast will not be possible, and the common time scale can only be achieved through a distributed synchronization. The exchange of packets carrying time stamps [1] may suffer from random delays in the construction and processing of the packet. Alternative methods based on the wireless coupling of the oscillators in the network nodes [1]-[12] have been proposed. In pulsecoupled oscillators [6], [8]-[9], pulsed signals are transmitted and the nodes must detect the time of arrival of these pulses [1]-[2]. Two different time scales are involved [5]: the fast one corresponding to the short-duration pulse and the slow one corresponding to the phase evolution of each of the oscillator elements. Due to the existence of two different time scales, the system is analyzed in terms of maps, by means of discretetime models [3]. On the other hand, in analogue coupling [1], [10]-[12] each node transmits a signal proportional to its local oscillation, with all the nodes transmitting and receiving continuously at the same time [1]-[3]. The analogue coupling is also known as diffusive [5]; this meaning that there is an interaction between the inherent damping of the oscillator elements and the coupling. Continuous models are used, unlike the case of pulse coupling, which enables a more insightful analysis of the system behavior. The previous works [5], [13] have demonstrated that the two synchronization mechanisms exhibit significant analogies in the limit of weak coupling.

Although there are many previous works on wireless coupled oscillators [1]-[13], most of them rely on ideal models [2]-[3] of both the oscillator circuits, represented in terms of phase variables, and the coupling action, described with constant scalar coefficients and time delays. The work [14] presented a first detailed investigation of a system of three wireless-coupled oscillators, using a formulation that relies on linearized oscillator elements, extracted from harmonicbalance (HB) simulations, as well as detailed descriptions of the coupling effects, which consider the antenna gain and signal propagation. The oscillator elements behave in an injection-locked mode, instead of being phase-locked loops (PLL). In the PLL case [2], the antenna signals enter the phase-detector of each oscillator and there is no clear solution to use a single antenna for both transmission and reception. This is easily achieved in the case of injection-locked operation, which should enable a simple testing of different network topologies. In fact, this work extends the one in [14] by considering a variety of oscillation configurations, which are inspired by the ones commonly employed in sensor networks [3], [7]-[8]. The topologies analyzed correspond to 
ring, fully-connected and star configurations, which will be investigated for different numbers of oscillator elements.

There are several works on coupled-oscillators systems intended for power combination and beam steering [15]-[26]. Additionally, references [27]-[28] present a novel interesting application for oscillator tuning via a variable delay using a loop of unidirectional oscillators. On the other hand, wireless systems generally involve the global coupling of a set of symmetrically distributed oscillators [1]-[5]. This is different from the beam-steering application, where the detuning of the peripheral oscillators [15]-[24] leads to different operation conditions in each oscillator. The coupling networks used for beam steering usually consist of a transmission line bounded by resistors. The system parameters are the bias voltages of the peripheral oscillators and the coupling coefficients only exhibit small changes, due to the variation of the oscillation frequency [21]-[22]. In the wireless case, the most relevant parameters are the distances between the oscillator elements, which must be suitably chosen to prevent instability. The magnitude of the coupling coefficients is affected by the basic propagation loss and the phase shift exhibits a periodic or quasi-periodic variation versus distance. The frequency dependence of the coupling effects has a relevant impact on the stability properties, unlike what is usually observed in beam-steering applications.

This work will consider the most common geometries of wireless-coupled systems. As will be shown, in symmetric topologies there exist several oscillation modes, depending on the number of elements, which lead to particular phase and amplitude distributions between the oscillator elements. The complete set of modes associated with a particular network topology is derived from an eigenvalue/eigenvector calculation [29] of an equivalent admittance matrix, accounting from the wireless-coupling effects. Then, the system behavior is analyzed in two manners. The first one is based on an analytical formulation that depends on the linearized HB models of the oscillator elements and the eigenvalue associated with each particular mode. This formulation will allow an efficient tracing of the complete solution curves (including multi-valued sections) versus any relevant parameter, such as the distance between the oscillator elements or the antenna gain. The second one will be based on a full nonlinear simulation at circuit level in HB. As will be shown, an initial identification of the modes enables a reduced simulation of the whole coupled topology using only one or two oscillator elements. Despite the higher accuracy of the full circuit-level simulation based on $\mathrm{HB}$, the analytical formulation is of high interest due to its capability to provide multi-valued sections of the solution curves in a straightforward manner.

The paper is organized as follows. Section II presents the general formulation used for the analysis of wireless-coupled oscillators. Section III describes the case of a ring topology. Section IV and Section V describes the cases of a fullyconnected and a star topology, respectively. Section VI presents the general stability analysis formulation. Section VII presents a study of the impact of discrepancies between the oscillator elements. Section VIII addresses the analysis of phase noise.

\section{General SyStem Formulation}

The wireless-coupled oscillators system will be composed of $N$ oscillator elements, so that each of the $N$ oscillators is injection locked by the signal transmitted by two or more system elements [1]-[13]. It is assumed that the oscillator system operates in a synchronized manner at the frequency $\omega_{s}$. Each oscillator is loaded with an antenna, exhibiting the radiation resistance $R_{r}$. Thus, the power transmitted by each oscillator will be $\left|V_{\text {out }}\right|^{2} /\left(2 R_{r}\right)$, where $V_{\text {out }}$ is the oscillator output voltage. On the other hand, the available power at reception can be expressed in terms of an equivalent injectioncurrent source $I_{s}$, connected in parallel at the output of the oscillator circuit [30]-[31]. The available power in a given oscillator $k$ due to the signal transmitted by the oscillator $m$ is:

$$
\frac{R_{r}\left|I_{s, m, k}\right|^{2}}{8}=\frac{V_{\text {out }, m}^{2}}{2 R_{r}} \frac{G_{t, m} G_{r, k} \lambda^{2}}{\left(4 \pi d_{m, k}\right)^{2}}
$$

where $G_{t, m}, G_{r, k}, d_{m, k}$ and $\lambda$ are, respectively, the transmitter and receiver antenna gains, the distance between the oscillators $m$ and $k$ and the wavelength at the oscillator operation frequency $\omega_{s}$. Solving for $\left|I_{s, m, k}\right|$, one obtains:

$$
\left|I_{s, m, k}\right|=\frac{C}{\omega_{s} R_{r} d_{m, k}} \sqrt{G_{t, m} G_{r, k}} V_{\text {out }, m}
$$

where $c$ is the speed of light. On the other hand, for the distance $d_{m, k}$, the phase shift due to propagation effects will be: $-\alpha=-\omega_{s} d_{m, k} / c$. Then, it will be possible to express the synchronizing input current at the oscillator $k$, due to the oscillator element $m$, as: $I_{s, m, k}=C_{m, k}\left(\omega_{s}, G_{\text {tot }}, d_{m, k}\right) V_{\text {out }, m}$, where the complex transconductance coefficient $C_{m, k}$ is:

$$
C_{m, k}\left(d_{m, k}, \omega_{s}\right)=\frac{c}{\omega_{s} R_{r} d_{m, k}} \sqrt{G_{t o t, m, k}} e^{-j \frac{d_{m, k}}{c} \omega_{s}}
$$

and $G_{t o t, m, k}=G_{t, m} G_{r, k}$ is the antenna gain product. The voltage controlled current sources modeling the coupling action between two particular oscillators elements $(k$ and $m)$ are represented in Fig. 1(a). The radiation resistance $R_{r}$ (usually $50 \Omega$ ) is absorbed into the oscillator circuit. For compactness, the complex transconductance function will be expressed in terms of magnitude and phase as follows:

$$
\begin{aligned}
& C_{m, k}=A_{m, k}\left(\omega_{s}, d_{m, k}\right) e^{-j \alpha_{m, k}\left(\omega_{s}, d_{m, k}\right)} \\
& \alpha_{m k}\left(\omega_{s}, d_{m k}\right)=\omega_{s} d_{m, k} / c
\end{aligned}
$$

There will be a phase change of $2 \pi$ radians for a distance variation of $\Delta d=1 /\left(3.33310^{-9} f\right)$. On the other hand, the rate of phase variation with frequency increases with the distance $d$. As an example, for a distance of $3 \mathrm{~m}$, the phase changes a whole interval of $2 \pi$ under a frequency change $\Delta f=100 \mathrm{MHz}$. 
In free-running conditions each oscillator will exhibit an amplitude and frequency given by $V_{m o}, \omega_{m o}$, where $m=1$ to $N$. In this free-running operation, the current to voltage ratio $Y$ is equal to zero at any node, and in particular, at the antenna connection node $\left(Y_{m}=0\right)$. When introduced into the network, the functions $Y_{m}$ will undergo a variation due to the current induced by the signals received from other oscillator elements, so it will no longer be zero. Assuming that all the oscillator elements are identical, two cases will be analyzed here. The first one considers a nonlinear dependence $Y\left(V_{m}, \omega_{5}\right)$, equal for all the $N$ elements, where $V_{m}(m=1$ to $N)$ is the oscillation amplitude. The second one is based on a linearization of the admittance function $Y$ about the free-running solution $V_{o}, \omega_{o}$, identical for all the oscillator elements [21]-[24], [32].

Under wireless-coupled conditions and assuming a synchronized behavior at the frequency $\omega_{s}$, the system can be formulated in a compact manner as:

$$
\left(\left[Y\left(\bar{V}, \omega_{s}\right)\right]-[C]\right) \bar{V}_{s \phi}=0
$$

where $\left[Y\left(\bar{V}, \omega_{s}\right)\right]$ is a diagonal matrix containing the nonlinear admittance function of the individual oscillators, $[C]$ is the coupling matrix, with the elements $C_{m, k}$ given in (3) -(4), and $\bar{V}_{s \phi}$ is the vector containing the oscillator voltages $V_{m} e^{j \phi_{m}}$ at the antenna nodes. Due to the system autonomy, the phase of one of the oscillator elements can be arbitrarily set to zero $\left(\phi_{1}=0\right)$. Critical parameters for the phase-shift distribution of the wireless system will be the distances between the oscillator elements, as gathered from (3)-(4).

To get analytical insight, a linearized version of (5) will also be considered. This will be valid under small induced currents, so that it is possible to perform at Taylor series expansion of the functions $Y\left(V_{m}, \omega_{s}\right)$ about the free-funning solution, with $Y=0$. Assuming, as stated, identical oscillator elements, with the free-running amplitude $V_{o}$ and free-running frequency $\omega_{o}$, each element of the linearized diagonal matrix $[Y(\bar{V})]$ is given by: $Y_{v} \Delta V_{m}+Y_{\omega} \Delta \omega_{s}$, where $\Delta V_{m}=V_{m}-V_{o}$ and $\Delta \omega_{s}=\omega_{s}-\omega_{0}$. The quantities $Y_{V}$ and $Y_{\omega}$ refer to the derivatives of the admittance function $Y$ (at the antenna connection node), calculated with respect to the amplitude and frequency. The derivatives are extracted from a HB simulation of the elementary oscillator, using one auxiliary generator (AG) [33]-[35], as shown in Fig. 1(b). They are obtained by applying finite differences to the $\mathrm{AG}$ amplitude and frequency [32].

In the following, the general equation (5) will be particularized to three different types of oscillator networks, with ring, fully-connected and star topologies. The individual oscillator element is the same in all cases [Fig. 1(b) and (c)]. It is built on the substrate Rogers $4003 \mathrm{C}$ and based on the transistor FET NE3210S01. The free-running frequency is $2.45 \mathrm{GHz}$ and the output power is $10 \mathrm{dBm}$.

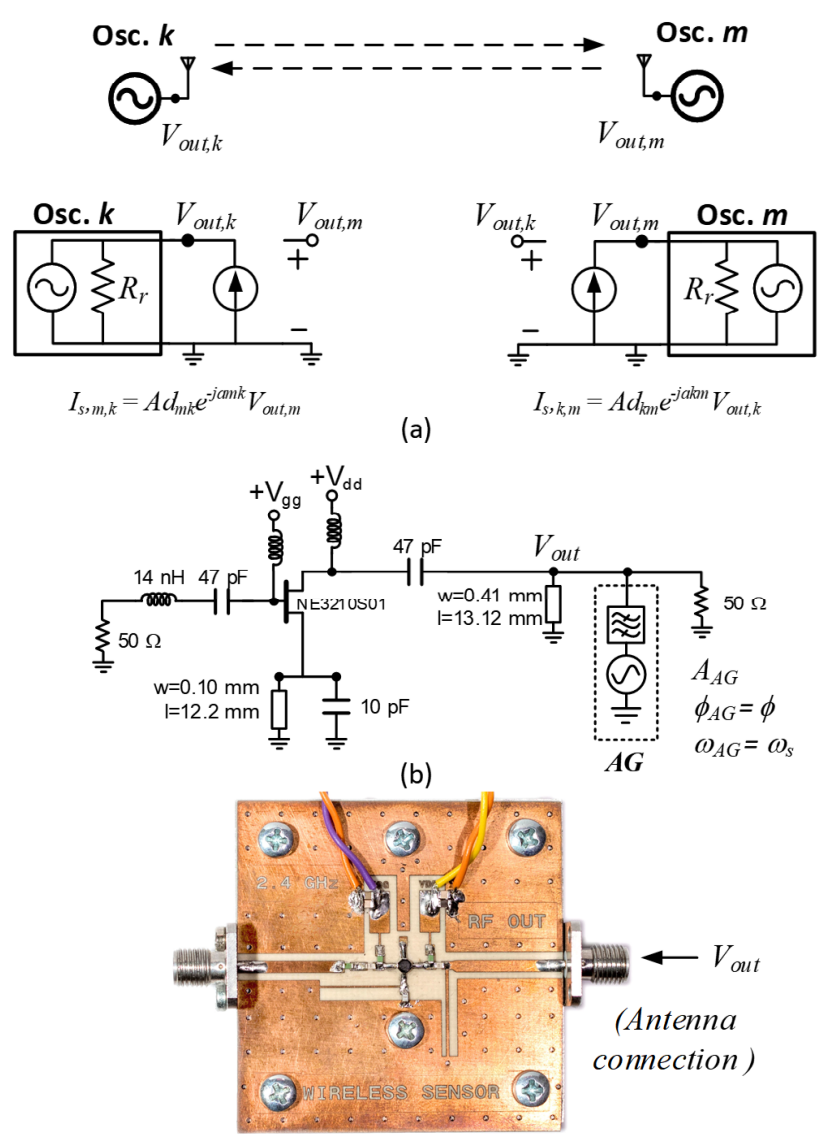

(c)

Fig. 1. Wireless coupling between oscillators $k$ and $m$. (a) Modelling of the coupling effects using voltage-controlled current sources. (b) Schematic of the oscillator based on the FET NE3210S01 operating at $2.45 \mathrm{GHz}$. To extract the linearized oscillator models from HB simulations an auxiliary generator (AG) is connected to the oscillator output node. (c) Prototype built on Rogers $4003 \mathrm{C}$.

\section{RING TOPOLOGY}

In a ring topology [3], [7], [8], [13], each node is connected to the two neighboring nodes of the network (Fig. 2). This topology, requiring directive antennas, is easy to install and reconfigure, but costly to manage, since the ring network can be disturbed by the failure of a single node. Nevertheless it constitutes a relatively simple initial case, very well suited for the illustration of the analysis method.

For identical distances between the oscillator elements and identical antenna gains, the transconductances $C_{m, k}$ should be equal and given by $C_{m, k}=A e^{-j \alpha}$. Then, the coupled network is governed by the matrix system (5), with the following coupling matrix: 


$$
\begin{aligned}
& {\left[\begin{array}{ccccc}
C_{11} & C_{12} & C_{13} & \cdots & C_{1 N} \\
C_{1 N} & C_{11} & C_{12} & \cdots & \vdots \\
\vdots & C_{1 N} & C_{11} & \cdots & \vdots \\
\vdots & \vdots & \vdots & \ddots & \vdots \\
C_{12} & C_{13} & \cdots & \cdots & C_{11}
\end{array}\right]=} \\
& {\left[\begin{array}{ccccc}
0 & A e^{-j \alpha} & 0 & \cdots & A e^{-j \alpha} \\
A e^{-j \alpha} & 0 & A e^{-j \alpha} & \cdots & 0 \\
0 & A e^{-j \alpha} & 0 & \cdots & 0 \\
\vdots & \vdots & \vdots & \ddots & \vdots \\
A e^{-j \alpha} & 0 & 0 & \cdots & 0
\end{array}\right]}
\end{aligned}
$$

In view of the system symmetry, one should expect solutions of (5) with identical amplitudes $V=V_{1}=V_{2}=\ldots=V_{N}$. Then, the possible oscillation modes are determined by the eigenvalues and eigenvectors of the matrix $[C]$. The eigenvalues of a circulant matrix are calculated as [29]:

$$
\lambda_{n}=C_{11}+C_{12} e^{j \frac{n 2 \pi}{N}}+C_{13} e^{j \frac{2 n 2 \pi}{N}}+\ldots+C_{1 N} e^{j \frac{(N-1) 2 \pi}{N}}
$$

where $n=0$ to $N-1$. The corresponding eigenvectors are:

$$
\bar{V}_{n}=\frac{V_{o}}{\sqrt{N}}\left(1 e^{j \frac{n 2 \pi}{N}} e^{j \frac{2 n 2 \pi}{N}} \ldots e^{j \frac{(N-1) 2 \pi}{N}}\right)^{T}
$$

The components of $\bar{V}_{n}$ are the voltages at the antenna connection nodes for the mode $n$, having a common value. The phase shift between adjacent nodes is given by $n 2 \pi / N$, which defines the distinct oscillation modes. When exciting the admittance-dimension matrix $[C]$ with a voltage vector agreeing with $\bar{V}_{n}$, all the nodes will exhibit the same equivalent admittance $\lambda_{n}$. Using (7), the eigenvalues of (6) can be written in the following compact form:

$$
\lambda_{n}=\zeta(n, N) A e^{-j \alpha}
$$

where $\zeta(n, N)$ is a scalar quantity that depends on the number $N$ of oscillators and the oscillation mode. The values of $\zeta(n, N)$ for $N=2$ to $N=8$ are shown in Table I. Note that for $N>2$ oscillators, the in-phase mode always corresponds to $\zeta=2$. The equivalent coupling current is given by $I_{n}=\lambda_{n} V$, where $V$ is node-voltage magnitude, identical for all the oscillator elements. As an example, for $N>2$, a distance $d=1$ $\mathrm{m}$, an oscillation frequency $f_{s}=2.4 \mathrm{GHz}$, equal antenna gains $G=5 \mathrm{~dB}$ and radiation resistance $R_{r}=50 \Omega$, the magnitude of the equivalent coupling admittance corresponding to the inphase mode is $\lambda_{0}=0.025 \Omega^{-1}$. For $N=5$, the magnitudes corresponding to the other modes are $\lambda_{72^{\circ}}=7.7810^{-4} \Omega^{-1}$ and $\lambda_{144^{\circ}}=0.002 \Omega^{-1}$. The modes with $90^{\circ}$ phase shift will see an equivalent input admittance equal to zero, so the coupled operation is impossible for this phase shift.

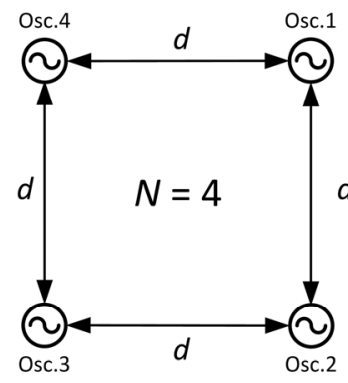

(a)

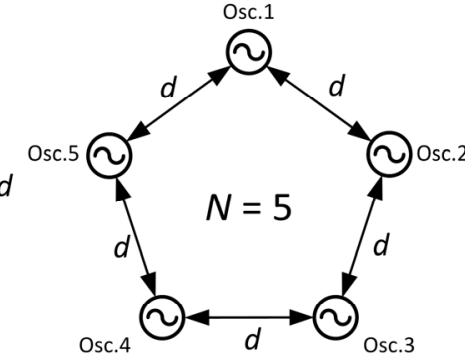

(b)

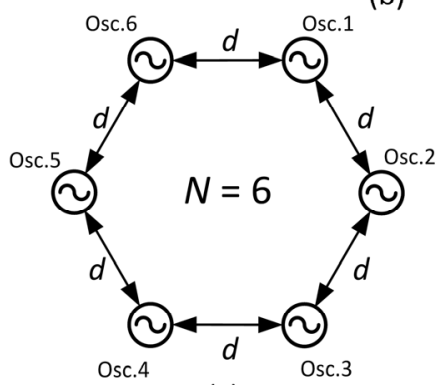

(c)

\begin{tabular}{|c|c|c|c|c|c|}
\hline$\overline{N N}$ & $\begin{array}{c}\text { In- } \\
\text { phase }\end{array}$ & Mode 2 & Mode 3 & "Mode 4 & "Mode 5 \\
\hline 2 & $1_{\left(0^{\circ}\right)}$ & $-1_{\left(180^{\circ}\right)}$ & & & \\
\hline 3 & $2\left(0^{\circ}\right)$ & $-1_{\left(120^{\circ}\right)}$ & & & \\
\hline 4 & $2_{\left(0^{\circ}\right)}$ & $0_{\left(90^{\circ}\right)}$ & $-2\left(180^{\circ}\right)$ & & \\
\hline 5 & $2\left(0^{\circ}\right)$ & $\begin{array}{l}\frac{-1+\sqrt{5}}{2} \\
\left(72^{\circ}\right)\end{array}$ & $\begin{array}{l}\frac{-1-\sqrt{5}}{2} \\
\left(144^{\circ}\right)\end{array}$ & & \\
\hline 6 & $2_{\left(0^{\circ}\right)}$ & $-1_{\left(120^{\circ}\right)}$ & $-2_{\left(180^{\circ}\right)}$ & $1_{\left(240^{\circ}\right)}$ & \\
\hline 8 & $22_{\left(0^{\circ}\right)}$ & $\sqrt{2}_{\left(45^{\circ}\right)}$ & $0_{\left(90^{\circ}\right)}$ & $\begin{array}{c}-2 \\
\left(180^{\circ}\right)\end{array}$ & $\begin{array}{l}-\sqrt{2} \\
\left(270^{\circ}\right)\end{array}$ \\
\hline
\end{tabular}

Fig. 2 Ring topology with different numbers $N$ of oscillator elements. Each oscillator is coupled by its closest neighbors.

TABLE I

VALUES OF $\zeta$ FOR DIFFERENT NUMBER OF OSCILLATORS AND MODES

Under the voltage excitation $\bar{V}_{n}$, the coupling effects at all the oscillator nodes can be described in terms of $\lambda_{n}$, so the whole system (5) can be replaced with a single complex equation. Splitting the total admittance function of each oscillator into real and imaginary parts $Y\left(V, \omega_{s}\right)=G\left(V, \omega_{s}\right)+j B\left(V, \omega_{s}\right)$, the oscillation condition for $N$ oscillator elements in the mode $n$ is:

$$
\begin{aligned}
& G\left(V, \omega_{s}\right)=\zeta(n, N) A\left(\omega_{s}, d\right) \cos \left[\alpha\left(\omega_{s}, d\right)\right] \\
& B\left(V, \omega_{s}\right)=-\zeta(n, N) A\left(\omega_{s}, d\right) \sin \left[\alpha\left(\omega_{s}, d\right)\right]
\end{aligned}
$$

Note that the phase shift between the oscillator elements is known beforehand and determined by the eigenvector(s) associated with $\lambda_{n}$, according to the Table I. This opens a new simple way to accurately simulate the whole coupled system in harmonic balance. Indeed, the analysis is performed by loading a single oscillator element with an equivalent admittance $Y_{e q}=-\lambda_{n}$, where $\lambda_{n}$ is given by (9). This frequency-dependent load implemented with an admittance box in commercial HB, as sketched in Fig. 3. Furthermore, 
provided there is information on the antenna behavior at higher frequencies, it will be possible to take into account coupling effects at higher harmonic terms $k \omega_{s}$. This will be done by loading the individual oscillator circuits with the harmonic values $Y_{e q, k}=-\lambda_{n, k}$, directly obtained by replacing $\omega_{s}$ with $k \omega_{s}$ in (9). The equivalent admittance(s) is defined in terms of the frequency variable. One should know the frequency dependence of the antenna gain in a wide frequency range, covering the harmonic frequencies. If the antenna gain function at the harmonic components is too low, one can use a function with negligible value from certain frequency.

The HB simulation of the single oscillator, loaded with $Y_{e q}=-\lambda_{n}$, is carried out with one auxiliary generator at the frequency $\omega_{A G}=\omega_{s}$, with an amplitude $A_{A G}$, which can be connected to an internal circuit node [32]-[35]. Because the phase shift between the oscillator elements is known beforehand, the single AG phase can be arbitrarily set to zero.

This described HB methodology has been applied to several coupled networks with $N=2$ to $N=8$, based on the oscillator circuit in Fig. 1. The common antenna gain is $G=5 \mathrm{~dB}$. The number of harmonic terms considered in the HB simulation is $N H=8$. All the modes existing for each $N$ have been analyzed and Fig. 4 presents the variation of the oscillation frequency $\omega_{s}$ versus the distance $d$. Note that a distinct solution curve exists for each oscillation mode. The results obtained with the reduced simulation, using a single oscillator, are overlapped with those obtained when simulating the full system of $N$ oscillators, which is computationally demanding. In fact, the circuit-level analysis of the entire coupled system requires the use of $N$ auxiliary generators [25]-[26] to sustain the $N$ individual oscillations. If no $\mathrm{AG}$ is connected to a particular oscillator element, this element will converge towards a nonoscillatory solution, which just responds to the signals entering through the equivalent coupling network. On the other hand, unless a proper initial value is given to the AG phases, no convergence is obtained in the optimization process used to solve (5) in HB. The AG phases must be set according to the phase distributions predicted by the eigenvalue/eigenvector analysis in (7)-(8).

\section{Passive admittance function \& Auxiliary Generator

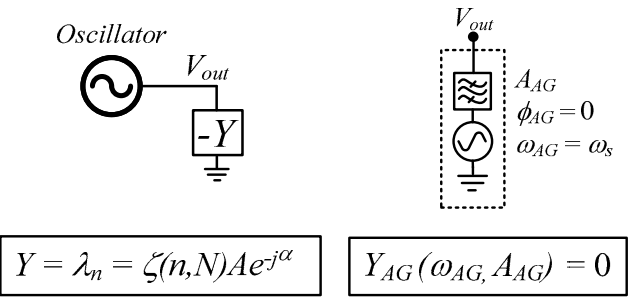

Fig. 3 Methodology for the simulation of the coupled system in a commercial HB simulator. This is based on the use of an equivalent coupling-admittance model, $Y_{e q}=-\lambda_{n}$. The eigenvalue $\lambda_{n}$ should be the one corresponding to the analyzed mode. The auxiliary generator used for this oscillator analysis is indicated. It can be connected to any internal node.

The results of the multi-oscillator circuit-level simulation (with $N$ AGs) are overlapped with those provided by the single-element simulation, as shown in Fig. 4. However, one should note that the (overlapped) curves calculated with HB are incomplete, which is due to the presence of turning points, or points with infinite slope. Passing through these turning point requires a demanding parameter switching [33]-[34], performed in a manual way. This has applied in Fig. 4 only for the specific cases $N=2\left(0^{\circ}\right)$ and $N=3\left(120^{\circ}\right)$. Lack of convergence was also experienced at many instances.

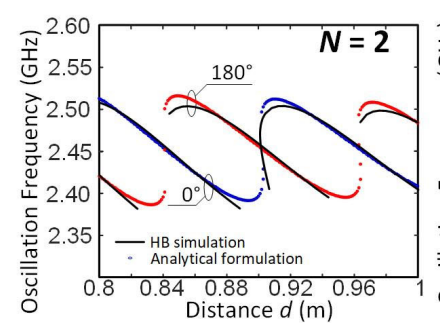

(a)

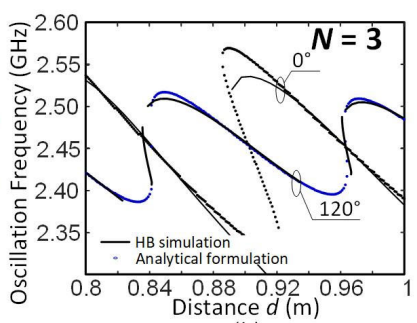

(b)
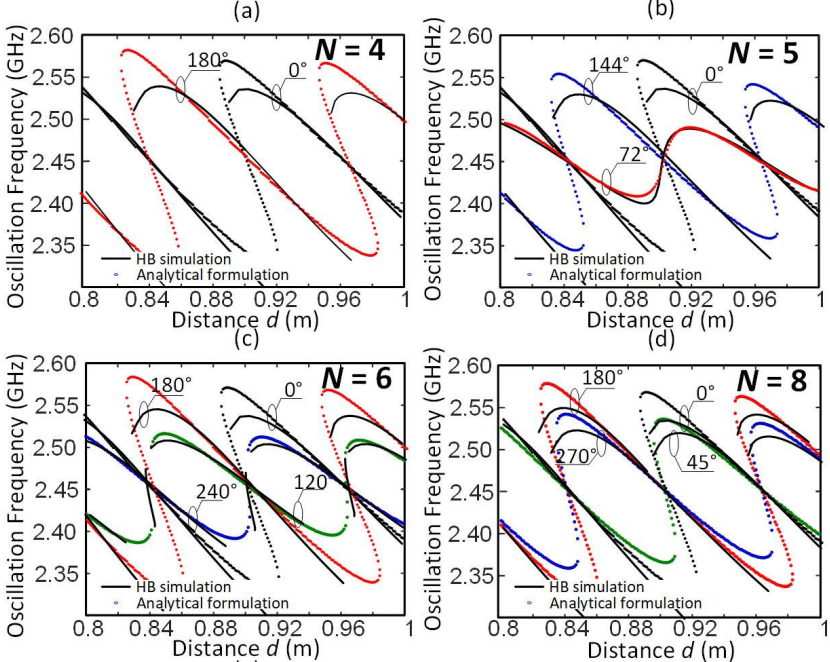

(e)

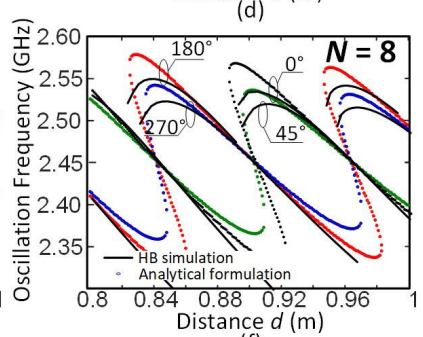

(f)

Fig. 4. Ring topology. Variation of the synchronized-oscillation frequency for the different oscillation modes versus the distance $d$, with for $N=2$ to 8 oscillator elements. The results two different circuit-level HB simulations and the semi-analytical formulation are compared

The described HB-simulation method provides a good accuracy but little insight into the system behavior, worsened by the impossibility to complete the solution curves. For a better understanding, it will be useful to perform an approximate calculation of the solutions of system (10). This will be based on a semi-analytical formulation, relying on the linearization of the individual-oscillator admittance function about the free-running regime, discussed in Section II. Applying this linearization to $G\left(V, \omega_{s}\right)$ and $B\left(V, \omega_{s}\right)$ in (10), one obtains the real-equation system:

$$
\begin{aligned}
& G_{v} \Delta V+G_{\omega} \Delta \omega_{s}=\zeta(n, N) A\left(\omega_{s}, d\right) \cos \left[\alpha\left(\omega_{s}, d\right)\right] \\
& B_{v} \Delta V+B_{\omega} \Delta \omega_{s}=-\zeta(n, N) A\left(\omega_{s}, d\right) \sin \left[\alpha\left(\omega_{s}, d\right)\right]
\end{aligned}
$$

where the subindex indicates the variable with respect to which the function is differentiated and higher-order amplitude increments have been neglected. Variations of the frequency and amplitude corresponding to the mode $n$ of the $N$-oscillator system are obtained by solving (11) for $\omega_{s}$ and $V$, which provides the two following decoupled scalar equations: 
$\omega_{\mathrm{s}}-\omega_{o}=$

$\frac{-\zeta(n, N) A\left(\omega_{s}, d\right)\left(G_{v} \sin \left[\alpha\left(\omega_{s}, d\right)\right]+B_{v} \cos \left[\alpha\left(\omega_{s}, d\right)\right]\right)}{\left(G_{v} B_{\omega}-G_{\omega} B_{v}\right)}$

$V-V_{o}=$

$\frac{\zeta(n, N) A\left(\omega_{s}, d\right)\left(B_{\omega} \cos \left[\alpha\left(\omega_{s}, d\right)\right]+G_{\omega} \sin \left[\alpha\left(\omega_{s}, d\right)\right]\right)}{\left(G_{v} B_{\omega}-G_{\omega} B_{v}\right)}$

The first equation is nonlinear in $\omega_{s}$, and must be numerically resolved, which has been done here through a simple zero-detection technique. Once $\omega_{\mathrm{s}}$ is known, the amplitude in coupled conditions is directly obtained from (b). The right side of (12) exhibits a double dependence on $\omega_{s}$, sinusoidal and inverse $\left(1 / \omega_{s}\right)$, which should lead to multivalued solutions for some parameter values, in agreement with the HB results in Fig. 4. For a given oscillator design, antenna gain and distance, the solution curve (in terms of $\omega_{s}$ or $V)$, associated to each mode, only depends on $\zeta(n, N)$. From the inspection of Table I, the in-phase solution will always exhibit the same values of $\omega_{s}$ and $V$ versus the distance $d$ (or any other parameter), no matter the number $N$ of oscillator elements. This is because each oscillator only receives injection signals from its two adjacent elements. Modes having the same frequency and amplitude no matter the value $N$ are shadowed in grey in Table I.

Fig. 4 presents a comparison of the results of the semianalytical formulation (11) with the circuit-level HB simulation. There is a very good agreement except at large excursions of the oscillation frequency, where the linearization of the admittance function about the free-running point becomes less accurate. Even though the HB analysis is more precise, the semi-analytical formulation has the advantage of easily predicting the multi-valued sections of the solution curves.

Special attention will be paid to the case of $N=5$ oscillator elements, which will also be studied in detail when addressing the other two topologies. The mode with $2 \pi / 5=72^{\circ}$ phase shift between the oscillator elements is single valued versus the distance $d$. However, the modes with $0^{\circ}$ and $2 \times 2 \pi / 5=144^{\circ}$ phase shift are multi-valued versus $d$. This is because the nonlinearity with respect to $\omega_{s}$ is more pronounced for higher values of the parameter $\zeta$, which is associated with stronger coupling effects. The respective values are $\zeta=2$ for $0^{\circ}$, $\zeta=-1.618$ for $144^{\circ}$ and $\zeta=0.6180$ for $72^{\circ}$. Due to the negative sign of $\zeta$ for $144^{\circ}$, the solution curve is in phase opposition with respect to the other two. As $d$ increases, the three curves in Fig. 4 tend to the free-running value $f_{o}=2.45$ $\mathrm{GHz}$, as should be the case for any $n$ and $N$. When approaching this value, coupling effects should be too weak to preserve the synchronized solutions against noise and perturbations. The in-phase mode is the one exhibiting the strongest coupling effects, so it can be maintained for a bigger d.

\section{Fully ConNeCted Topology}

In a fully connected topology [8], [36], each oscillator is wireless-coupled to all the rest of oscillators in the network. A spatial distribution based on regular polygons (Fig. 5) is usually considered, so that the diagonals are related with the side $d$ of the polygons. Each oscillator will have the same relative position with respect to the other $N-1$ elements, and the symmetries will give rise to a number of distinct distance values, depending on the number $N$. For instance, with $N=4$ oscillator elements, there are two different distance values: $d$ and $\sqrt{2} d$ [Fig. 5(a)]. In the case of $N=5$ oscillator elements, there are also two different distance values, given by $d$ and $(1+\sqrt{5} / 2) d \equiv \delta d$ [Fig. 5(b)]. In the case of $N=6$, there are three different distance values: $d, 2 d \equiv \delta_{1} d$ and $\sqrt{3} d \equiv \delta_{2} d$ [Fig. 5(c)]. For higher $N$, the distances are also easily identified due to the regular system geometry. For illustration, the cases of $N=4$ to $N=6$ will be considered here.

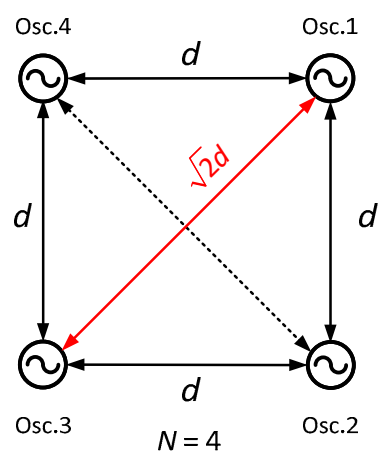

(a)

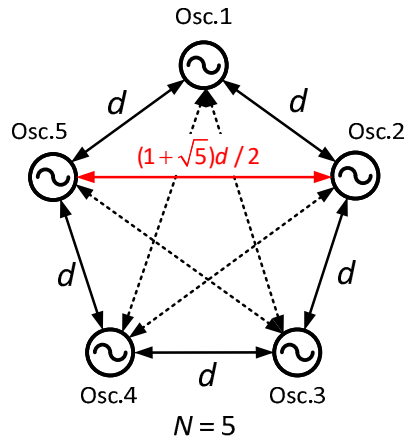

(b)

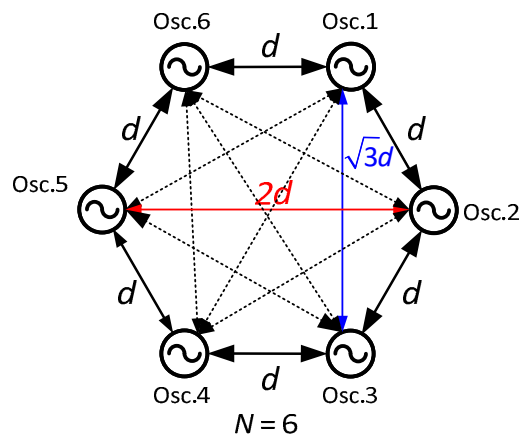

(c)

Fig. 5. Fully connected topology. Each oscillator is wireless-coupled to all the rest of oscillators in the network. (a) $N=4$. (b) $N=5$. (c) $N=6$.

\section{a) $\quad N=5$ oscillator elements}

With $N=5$, each oscillator is at a distance $d$ from its adjacent elements and at a distance $\delta d$ from the rest of elements. The respective coupling transconductances are:

$$
\begin{aligned}
& C(d)=\frac{c}{\omega_{s} R_{r} d} \sqrt{G_{t o t}} e^{-j \frac{d}{c} \omega_{s}}=A e^{-j \alpha} \\
& C(\delta d)=\frac{c}{\omega_{s} R_{r} \delta d} \sqrt{G_{t o t}} e^{-j \frac{\delta d}{c} \omega_{s}}=\frac{A}{\delta} e^{-j \alpha \delta}
\end{aligned}
$$

where the magnitude and phase of the transconductance for 
$\delta d$ has been related with the one corresponding to $d$. The coupling matrix $[C]$ is:

$$
[C]=\left[\begin{array}{ccccc}
0 & A e^{-j \alpha} & \frac{A}{\delta} e^{-j \alpha \delta} & \frac{A}{\delta} e^{-j \alpha \delta} & A e^{-j \alpha} \\
A e^{-j \alpha} & 0 & A e^{-j \alpha} & \frac{A}{\delta} e^{-j \alpha \delta} & \frac{A}{\delta} e^{-j \alpha \delta} \\
\frac{A}{\delta} e^{-j \alpha \delta} & A e^{-j \alpha} & 0 & A e^{-j \alpha} & \frac{A}{\delta} e^{-j \alpha \delta} \\
\frac{A}{\delta} e^{-j \alpha \delta} & \frac{A}{\delta} e^{-j \alpha \delta} & A e^{-j \alpha} & 0 & A e^{-j \alpha} \\
A e^{-j \alpha} & \frac{A}{\delta} e^{-j \alpha \delta} & \frac{A}{\delta} e^{-j \alpha \delta} & A e^{-j \alpha} & 0
\end{array}\right]
$$

The coupling matrix is still a circulant one. Due to the system symmetry, one can expect all the oscillators to exhibit the same amplitude, so the oscillation modes are defined by the eigenvalues/eigenvectors of $[C]$, calculated from (7) and (8). After some manipulation, the eigenvalues of (14) can be written in the following general form:

$$
\lambda_{n}=\beta(n) A e^{-j \alpha}+\gamma(n) \frac{A}{\delta} e^{-j \alpha \delta}
$$

where the coefficients $\beta(n)$ and $\gamma(n)$ are given in Table II. In turn, the phase distributions of the various modes are defined by the vector (8). As in the case of the ring topology, the HB circuit-level simulation will be carried out with a single oscillator element, loaded with the equivalent (frequency-dependent) admittance $Y_{e q}=-\lambda_{n}$. As an example, for $N=5$, a distance $d=1 \mathrm{~m}$, an oscillation frequency $f_{s}=2.4 \mathrm{GHz}$, equal antenna gains $G=5 \mathrm{~dB}$ and radiation resistance $R_{r}=50 \Omega$, the magnitude of the equivalent coupling admittance corresponding to the in-phase mode is $\lambda_{0}=0.004 \Omega^{-1}$. The magnitudes corresponding to the other modes are $\lambda_{72^{\circ}}=5.9110^{-4} \Omega^{-1}$ and $\lambda_{144^{\circ}}=0.0016 \Omega^{-1}$. These magnitudes are smaller than in the ring topology due to the interaction of the two complex exponential terms, with an incommensurate ratio $\delta=(1+\sqrt{5}) / 2$. To get insight into the system behavior, a semi-analytical formulation will also be derived, linearizing the individual-oscillator admittance function $Y\left(V, \omega_{s}\right)$ about the free-running point. The oscillation condition at each mode $n$ is given by:

$$
Y_{v} \Delta V+Y_{\omega} \Delta \omega=\beta(n) A e^{-j \alpha}+\gamma(n) \frac{A}{\delta} e^{-j \alpha \delta}
$$

Splitting (16) into real and imaginary parts and solving for $\omega_{s}$ and $V$, one obtains two decoupled scalar equations:

$$
\begin{aligned}
& \Delta \omega_{s}=\omega_{s}-\omega_{o}= \\
& \frac{A\left(\omega_{s}, d\right)}{G_{v} B_{\omega}-G_{\omega} B_{v}}\left\{\begin{array}{l}
-G_{v}\left[\beta(n) \sin \alpha+\frac{\gamma(n)}{\delta} \sin (\delta \alpha)\right] \\
-B_{v}\left[\beta(n) \cos \alpha+\frac{\gamma(n)}{\delta} \cos (\delta \alpha)\right]
\end{array}\right\} \text { (a) } \\
& \Delta V=V-V_{o}= \\
& \frac{A\left(\omega_{s}, d\right)}{G_{v} B_{\omega}-G_{\omega} B_{v}}\left\{\begin{array}{l}
G_{\omega}\left[\beta(n) \sin \alpha+\frac{\gamma(n)}{\delta} \sin (\delta \alpha)\right] \\
+B_{\omega}\left[\beta(n) \cos \alpha+\frac{\gamma(n)}{\delta} \cos (\delta \alpha)\right]
\end{array}\right\}
\end{aligned}
$$

The oscillation frequency and amplitude exhibit double dependence on $d$ : inversely proportional, due to the coefficient $A\left(\omega_{s}, d\right)$, and "quasi-periodic" due to the term in braces, with a non-rational relationship between the angles in the sinusoidal terms, given by $\delta=(1+\sqrt{5}) / 2$. To particularize the above expressions to the in-phase mode, one should make $\beta(0)=2$ and $\gamma(0)=2$. Fig. 6(a) presents the variations of $\omega_{s}$ versus $d$, corresponding to the in-phase mode, for $N=5$. Results of (17) are compared with those obtained through HB circuit-level simulations with $N H=8$ of the whole coupled system and a single element, which are overlapped. The accuracy of the analytical formulation degrades for relatively large excursions of the frequency and amplitude, which is due to the limitations of the oscillator-admittance linear model. On the other hand, the HB simulation is unable to provide the entire solution curves, as was the case with ring topology. Thus, the two kinds of analysis can be considered complementary.
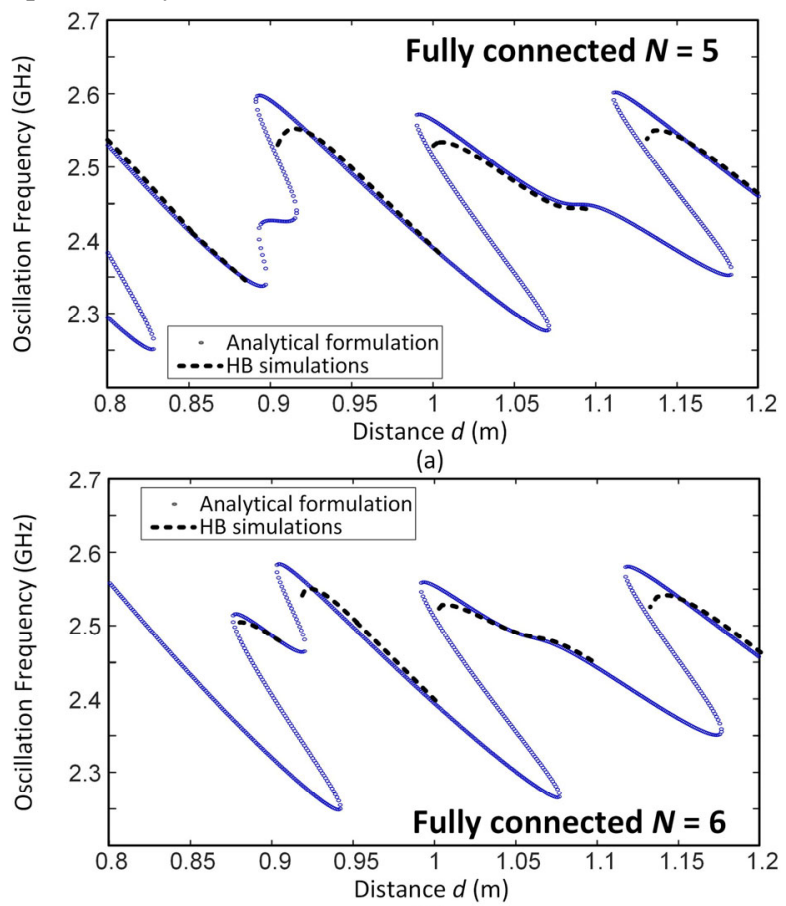

(b)

Fig. 6 Fully-connected topology with $G=5 \mathrm{~dB}$. Oscillation frequency of the in-phase mode versus the distance $d$. Results from the analytical formulation are compared with circuit-level HB simulations using multiple elements and a reduced system of a single element (overlapped). (a) $N=5$. (b) $N=6$. 


\section{b) Comparison of coupling effects for various} numbers of oscillator elements

Using the semi-analytical formulation under an arbitrary number of oscillators $N$, it is straightforward to derive the following expression for the oscillation frequency $\omega_{s}$ :

$$
\Delta \omega_{s}=\omega_{s}-\omega_{o}=\frac{A\left(\omega_{s}, d\right)}{G_{v} B_{\omega}-G_{\omega} B_{v}} \psi(n, N)
$$

where $\psi(n, N)$ is a function that depends on the oscillation mode $n$ and the number $N$. The coefficients $\psi(0, N)$ for $N=4$ to $N=6$ are given by:

$$
\begin{aligned}
& \psi(0,4)=\left\{\begin{array}{l}
-G_{v}\left[2 \sin \alpha+\frac{1}{\sqrt{2}} \sin (\alpha \sqrt{2})\right] \\
-B_{v}\left[2 \cos \alpha+\frac{1}{\sqrt{2}} \cos (\alpha \sqrt{2})\right]
\end{array}\right\} \\
& \psi(0,5)=\left\{\begin{array}{l}
-G_{v}\left[2 \sin \alpha+\frac{2}{1+\sqrt{5}} \sin (\alpha(1+\sqrt{5}) / 2)\right] \\
-B_{v}\left[2 \cos \alpha+\frac{2}{1+\sqrt{5}} \cos (\alpha(1+\sqrt{5}) / 2)\right]
\end{array}\right\} \\
& \psi(0,6)=\left\{\begin{array}{l}
-G_{v}\left[2 \sin \alpha+\frac{2}{\sqrt{3}} \sin (\sqrt{3} \alpha)+\frac{1}{2} \sin (2 \alpha)\right] \\
-B_{v}\left[2 \cos \alpha+\frac{2}{\sqrt{3}} \cos (\sqrt{3} \alpha)+\frac{1}{2} \cos (2 \alpha)\right]
\end{array}\right.
\end{aligned}
$$

Fig. 6(b) presents the variation of the oscillation frequency $\omega_{s}$, corresponding to the in-phase mode, traced versus $d$ for $N=6$ oscillator elements. Results of the analytical formulation are successfully compared with those obtained with circuitlevel HB simulations.

TABLE II

FULLY CONNECTED TOPOLOGY: VALUES OF $\beta, \gamma$ AND $\Delta \phi$ FOR $N=5$

\begin{tabular}{|c|c|c|c|c|c|}
\cline { 2 - 6 } \multicolumn{1}{c|}{} & $\lambda_{o}$ & $\lambda_{1}$ & $\lambda_{2}$ & $\lambda_{3}=\lambda_{2}$ & $\lambda_{4}=\lambda_{1}$ \\
\hline \hline $\boldsymbol{\beta}$ & 2 & $\frac{-1+\sqrt{5}}{2}$ & $\frac{-1-\sqrt{5}}{2}$ & $\frac{-1-\sqrt{5}}{2}$ & $\frac{-1+\sqrt{5}}{2}$ \\
\hline$\gamma$ & 2 & $\frac{-1-\sqrt{5}}{2}$ & $\frac{-1+\sqrt{5}}{2}$ & $\frac{-1+\sqrt{5}}{2}$ & $\frac{-1-\sqrt{5}}{2}$ \\
\hline \hline$\Delta \boldsymbol{\phi}$ & $\mathbf{0}^{\circ}$ & $\mathbf{7 2}^{\circ}$ & $\mathbf{1 4 4}^{\circ}$ & $\mathbf{2 1 6}^{\circ}=-\mathbf{1 4 4}^{\circ}$ & $\mathbf{2 8 8}^{\circ}=-\mathbf{7 2}^{\circ}$ \\
\hline \hline
\end{tabular}

\section{c) Experimental results}

Fig. 7 presents the measurement setup for the fully connected topology with $N=5$ oscillator elements. It is based on the use of an Agilent 90804A Digital Storage Oscilloscope and two E4446A PSA spectrum analyzers in order to visualize the oscillator waveforms, the synchronized spectrum and the phase noise simultaneously. Note that there were limitations in the lab size and equipment mobility to separate the oscillators a large distance, so measurements were carried out in the interval $d=1.1 \mathrm{~m}$ to $d=1.4 \mathrm{~m}$. To provide an estimation of the coupling capacity, when using an oscillator connected to an antenna as a transmitter, the power measured by connecting an identical antenna to a spectrum analyzer is $P_{c}=-27 \mathrm{dBm}$ at a distance of $1.4 \mathrm{~m}$. Fig. 8 (a) presents the measured in- phase waveforms, obtained for $d=1.2 \mathrm{~m}$. They were extracted at the gate terminals. The in-phase mode was not stable for all the distance values. For $d=1.28 \mathrm{~m}$, an oscillation mode with $144^{\circ}$ phase shift between adjacent elements was obtained instead, shown in Fig. 8(b). For clarity, only two waveforms are presented, though the same phase shift values were obtained between the any two other adjacent elements. In Fig. 9 the frequency measurements for the in-phase mode have been traced versus $d$, in the two cases of $N=4$ and $N=5$, where they can be compared with the analytical results. The vertical dashed lines indicate the stable ranges where the inphase mode could be measured. Outside these ranges, modes corresponding to other phase shifts and quasi-periodic solutions were obtained. Neither with $N=4$ nor with $N=5$ it was possible to obtain any measurements of the in-phase mode in the multi-valued interval of the solution curve. This is attributed to the fact that the distance was not varied continuously, since for each change of $d$, the full set-up has to be readjusted. From the above experimental results, one can gather that the stability analysis of these multi-oscillator systems is essential.

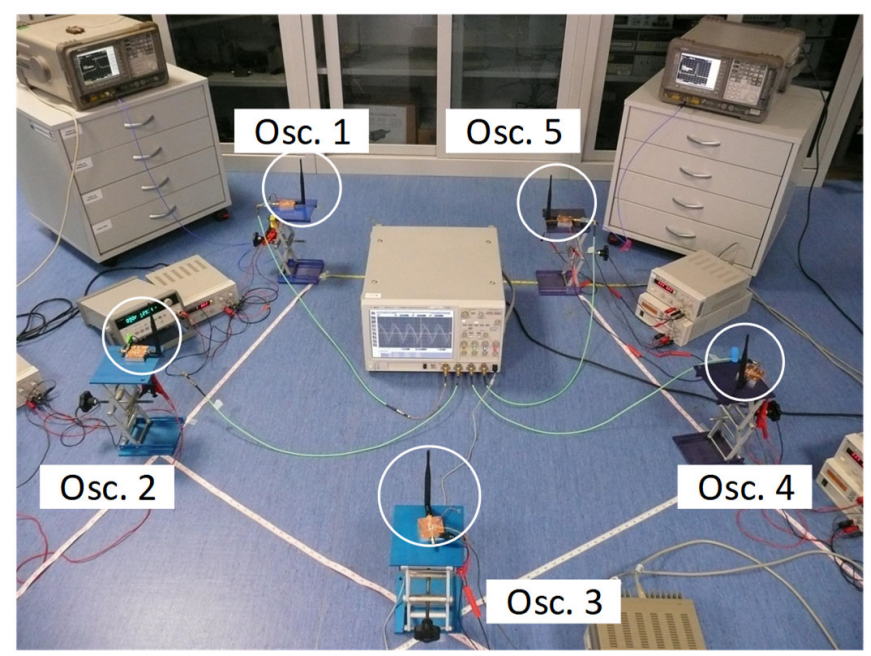

Fig. 7 Experimental characterization of the fully-connected topology. Measurement setup for fully connected topology of $N=5$. It is based on the use of an Agilent 90804A Digital Storage Oscilloscope and two E4446A PSA spectrum analyzers in order to visualize the oscillator waveforms, the synchronized spectrum and the phase noise simultaneously. 


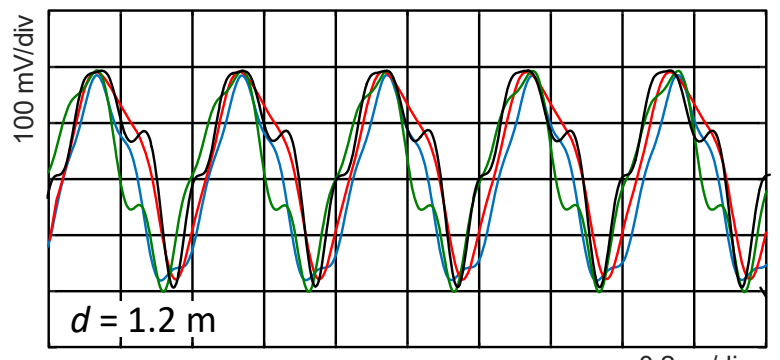

(a)

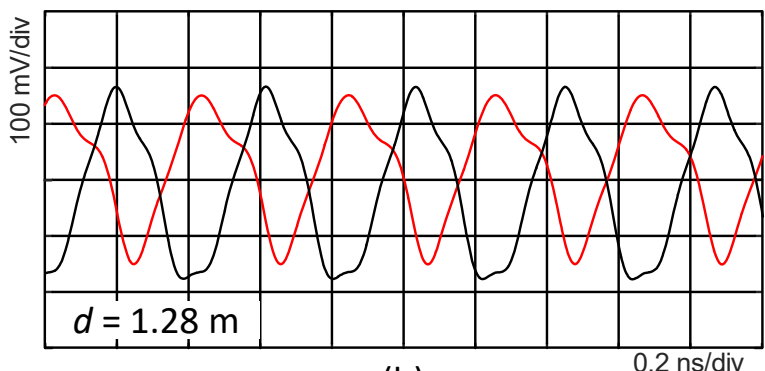

(b)

Fig. 8 Experimental characterization of the fully connected topology with $N=5$. Waveforms are measured at the gate terminals. (a) In-phase waveforms for $d=1.2 \mathrm{~m}$. (b) Waveforms with $144^{\circ}$ phase shift, for $d=1.28 \mathrm{~m}$.

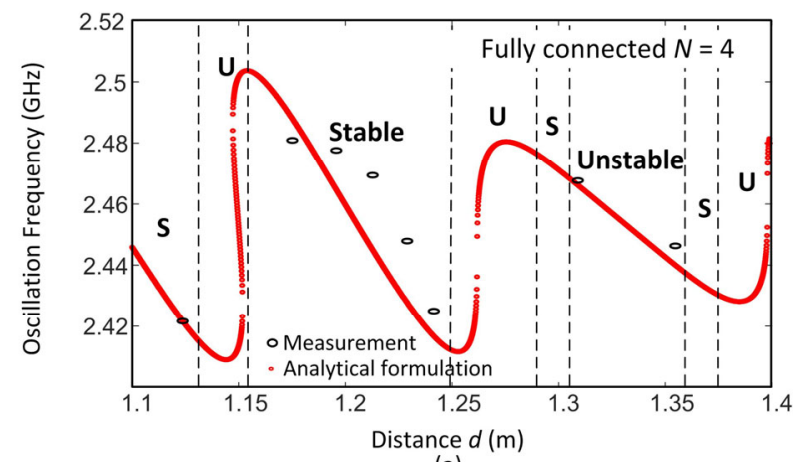

(a)

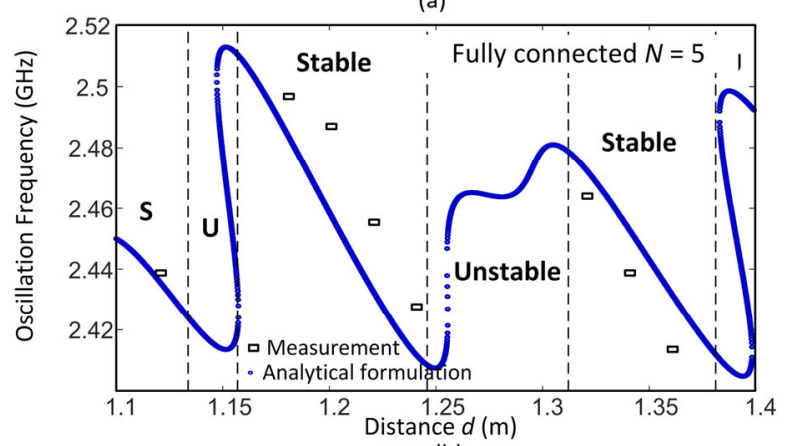

(b)

Fig. 9 Fully connected topology. Measurements of the oscillation frequency, corresponding to the in-phase mode, versus the distance $d$. They can be compared with the analytical predictions. The vertical dashed lines indicate the stable ranges where the in-phase mode could be measured. (a) $N=4$. (b) $N=5$.

\section{Star TOPOLOGY}

The star network topology (Fig. 10) is one of the most commonly employed in wireless networks [3]. It has several advantages, such as its simplicity and its ability to keep the remote node's power consumption at a minimum. It also allows low latency communications between the remote node and the base station. However, the base station must be within radio transmission range of all the individual nodes and it is not as robust as other networks due to its dependency on a single node to manage the network. The central node is coupled to the $N-1$ remaining oscillator elements, whereas each of these elements is coupled to the central node only. This will generally demand the use of an omnidirectional antenna in the central node and directive antennas in the other ones. In the usual case of a symmetric distribution of the $N-1$ peripheral oscillators about the central one, the system can be described in terms of two oscillators only, one corresponding to the central node and the other being representative of any of the remaining $N-1$ oscillator elements, in identical conditions. Taking the phase origin at the central node, the system equations are:

$$
\begin{aligned}
& Y\left(V_{1}, \omega_{s}\right) V_{1}=(N-1) A e^{-j \alpha} V_{2} e^{j \phi_{2}} \\
& Y\left(V_{2}, \omega_{s}\right) V_{2} e^{j \phi_{2}}=A e^{-j \alpha} V_{1}
\end{aligned}
$$

It depends on only two distinct oscillation amplitudes, $V_{1}$ for the central oscillator, and $V_{2}$, for the rest of oscillator elements, and one common phase shift $\phi_{2}$, as well as $\omega_{s}$. The above system can be nonlinearly solved in HB by using two oscillators connected through the following $2 \times 2$ couplingadmittance matrix:

$$
\left[Y_{c}\right]=\left[\begin{array}{cc}
0 & (N-1) A e^{-j \alpha} \\
A e^{-j \alpha} & 0
\end{array}\right]
$$

As an example, for $N=5$, a distance $d=1 \mathrm{~m}$, an oscillation frequency $f_{s}=2.4 \mathrm{GHz}$, equal antenna gains $G=5 \mathrm{~dB}$ and radiation resistance $R_{r}=50 \Omega$, the magnitude of the equivalent coupling admittance at the central oscillator is $(N-1) A=0.005 \Omega^{-1}$ and at the peripheral oscillators it is $A=0.0013 \Omega^{-1}$. The implementation of this coupling matrix is sketched in Fig. 11. The circuit level analysis will require two AGs, with the respective amplitudes $A_{A G 1}=V_{1}$ and $A_{A G 2}=V_{2}$ and phase values $\phi_{A G 1}=0$ and $\phi_{A G 2}=\phi_{2}$, both operating at the frequency $\omega_{A G}=\omega_{s}$. Two non-perturbation conditions $Y_{A G 1}=0$ and $Y_{A G 2}=0$ (one for each oscillator element) must be resolved simultaneously in terms of the four variables $A_{A G 1}, A_{A G 2}, \phi_{A G 2}$ and $\omega_{s}$. On the other hand, the semianalytical formulation, is directly obtained by introducing the linearization $Y_{v} \Delta V_{m}+Y_{\omega} \Delta \omega_{s}$, where $m=1,2$, into (20).

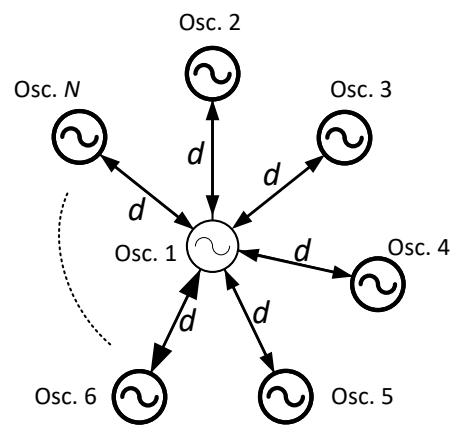

Fig. 10 Star topology. Each oscillator is coupled to a main oscillator (Osc.1) which, in turn, is coupled to all the network elements. 

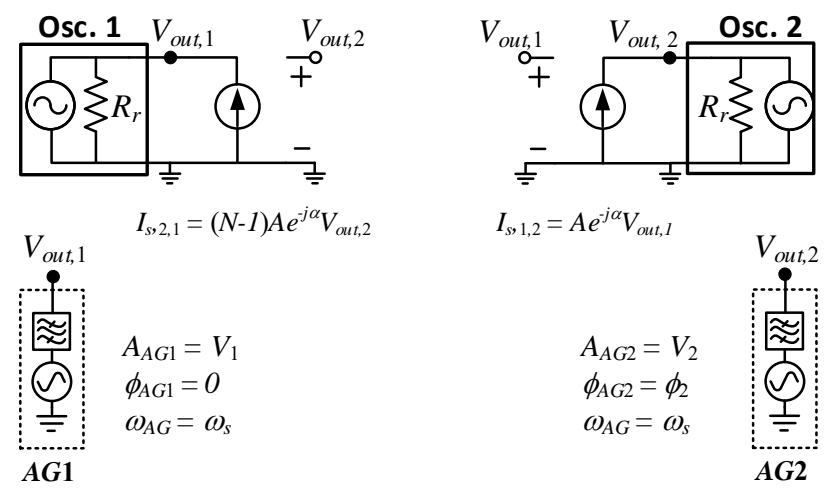

Fig. 11 Equivalent two-oscillator system used for the HB simulation of a network with a star topology. The auxiliary generators used for this simulation are indicated.

Fig. 12 presents a comparison of the results obtained through the semi-analytical formulation and with the HB circuit-level simulation, with similar behavior as in the previous cases. The oscillation frequency $\omega_{s}$ has been traced versus $d$ for $N=2$ and $N=4$. The number $N$ affects one of the elements coupling admittance matrix [see (21)]. For $N=2$, there are two distinct modes, corresponding to the in-phase solution and a solution with $180^{\circ}$ phase shift. This is because with $N=2$, the coupling matrix in (21) becomes a circulant matrix. However, for $N \neq 2$, the phase shift with respect to the central node varies continuously with $d$, since the admittance matrix is not symmetrical. Nevertheless, the peripheral oscillator elements should be in phase, as each one is connected only with the central node in a manner identical to the rest of oscillators. Measurement points are superimposed in the $d$ intervals with stable behavior.
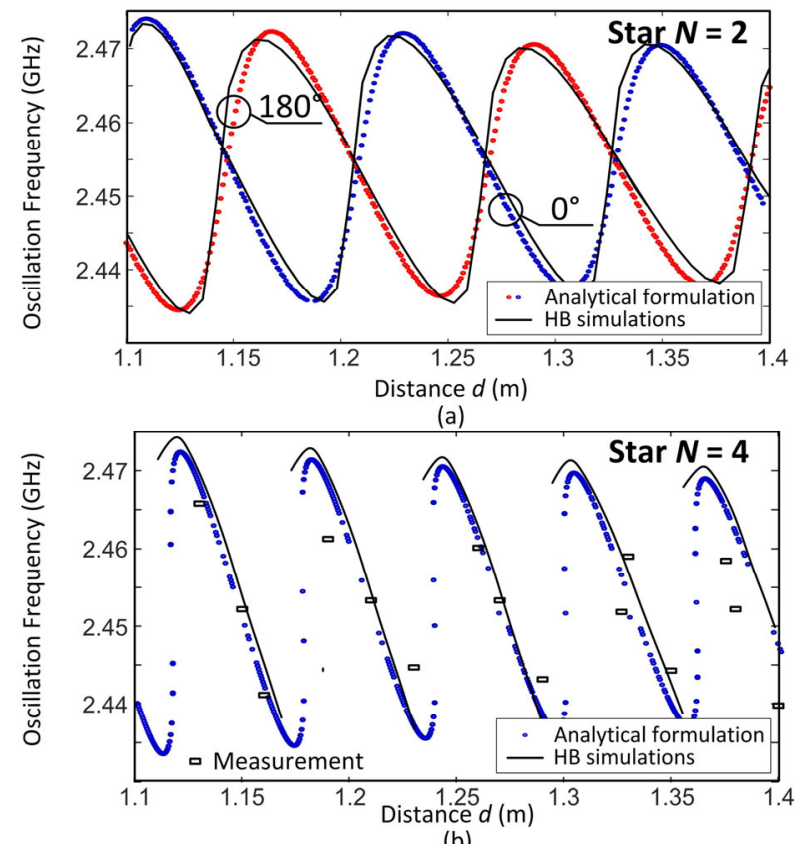

(b)

Fig. 12 Star topology. Comparison of the results obtained through the semianalytical formulation and the circuit level HB simulation using two oscillator elements. The oscillation frequency $\omega_{s}$ has been traced versus $d$. (a) $N=2$. Two modes, in-phase and with $180^{\circ}$ phase shift, are distinguished. (b) $N=4$. Measurement points are superimposed in the $d$ intervals with stable behavior.

\section{STABILITY ANALYSIS}

For compactness, any solution of the coupled system (5) will be expressed as the product: $\left[\exp \left(j \phi_{s, m}\right)\right]_{d} \bar{V}_{s}$, where the matrix on the left side is diagonal and contains exponential terms with $m$ going from 1 to $N$, and the vector $\bar{V}_{s}$ is composed of the oscillation amplitudes, ordered in the same manner. For the stability analysis a small perturbation of complex frequency $s$ is applied to this solution, which will give rise to small increments in the oscillator amplitudes and phases [15]-[26], represented in vector form as $\delta \bar{V}_{s}(t), \delta \bar{\phi}_{s}(t)$. Because the perturbation is small, the system can be linearized about the particular steady-state solution, which leads to the following system:

$$
\begin{aligned}
& \left\{\left[\frac{\partial Y\left(\bar{V}_{s}, \omega_{s}\right)}{\partial \bar{V}}\right]\left[V_{s, m} e^{j \phi_{s, m}}\right]_{d}+\left[Y\left(\bar{V}_{s}, \omega_{s}\right)\right]\left[e^{j \phi_{s, m}}\right]_{d}\right\} \delta \bar{V}_{s}(t)+ \\
& \left\{\left[Y\left(\bar{V}_{s}, \omega_{s}\right)\right]-[C]\right\}\left[j V_{s, m} e^{j \phi_{s, m}}\right]_{d} \delta \bar{\phi}_{s}(t)+ \\
& \left\{\left[\frac{\partial Y\left(\bar{V}_{s}, \omega_{s}\right)}{\partial j \omega}\right]-\left[\frac{\partial C\left(\omega_{s}\right)}{\partial j \omega}\right]\right\} s\left[e^{j\left(\phi_{s, m}+\delta \phi_{s, m}(t)\right)}\right]_{d}\left(\bar{V}_{s}+\delta \bar{V}_{s}(t)\right)=0
\end{aligned}
$$

The subindex $d$ indicates diagonal matrixes, with diagonal elements following the index $m$, which runs from 1 to $N$. In the wireless system, the coupling matrix exhibits significant frequency dependence, so its frequency derivative $\left[\partial C\left(\omega_{s}\right) / \partial j \omega\right]$ must be taken into account in the calculation. The increment $s$ acts like a time differentiator, so one can carry out the following substitutions [33], [37]:

$$
\begin{aligned}
& s\left[e^{j\left(\phi_{s, m}+\delta \phi_{s, m}(t)\right)}\right]_{d}\left(\bar{V}_{s}+\delta \bar{V}_{s}(t)\right) \cong \\
& {\left[V_{s, m} e^{j \phi_{s, m}}\right]_{d}\left[\frac{1}{V_{s, m}} \delta \dot{V}_{s, m}(t)+j \delta \dot{\phi}_{s, m}(t)\right]_{v}}
\end{aligned}
$$

where the subindex applied to the terms between brackets indicates a diagonal matrix $(d)$ or a vector $(v)$. Replacing (23) into (22), and neglecting the second-order terms affecting $\delta \bar{V}_{s}$ one obtains:

$$
\begin{aligned}
& \left\{\left[\frac{\partial Y\left(\bar{V}_{s}, \omega_{s}\right)}{\partial \bar{V}}\right]\left[V_{s, m} e^{j \phi_{s, m}}\right]_{d}\right\} \delta \bar{V}_{s}(t)+ \\
& \left\{\left[Y\left(\bar{V}_{s}, \omega_{s}\right)\right]-[C]\right\}\left[j V_{s, m} e^{j \phi_{s, m}}\right]_{d} \delta \bar{\phi}_{s}(t)+ \\
& \left\{\left[\frac{\partial Y\left(\bar{V}_{s}, \omega_{s}\right)}{\partial j \omega}\right]-\left[\frac{\partial C\left(\omega_{s}\right)}{\partial j \omega}\right]\right\}\left[V_{s, m} e^{j \phi_{s, m}}\right]_{d} \\
& {\left[\frac{1}{V_{s, m}} \delta \dot{V}_{s, m}(t)+j \delta \dot{\phi}_{s, m}(t)\right]_{V}=0}
\end{aligned}
$$

Assembling the components affecting the time-differentiated increments, system (24) can then be rewritten as:

$$
\left[M_{1}\right] \delta \dot{\bar{x}}=\left[M_{2}\right] \delta \bar{x}
$$

where $\delta \bar{x}$ is:

$$
\delta \bar{x}(t)=\left[\begin{array}{llllllll}
\delta V_{1} & \delta V_{2} & \ldots & \delta V_{N} & \delta \phi_{1} & \delta \phi_{2} & \ldots & \delta \phi_{N}
\end{array}\right]^{T}
$$

The above stability analysis can be applied in combination 
with a HB simulation of the reduced system. In fact, there are two matrixes containing the derivatives of the individual oscillator admittance functions:

$$
\left[\frac{\partial Y\left(\bar{V}_{s}, \omega_{s}\right)}{\partial \bar{V}}\right] ; \quad\left[\frac{\partial Y\left(\bar{V}_{s}, \omega_{s}\right)}{\partial j \omega}\right]
$$

The above matrixes can be obtained applying finite differences to the $\mathrm{AG}(\mathrm{s})$ used to obtain the steady-state solution of the reduced circuit, with only one oscillator or two oscillators, in the case of the star configuration. For each finite difference, the current-to-voltage ratio $Y$ is calculated, considering the current entering the oscillator, instead of the total current circulating through the AG. HB is used to obtain the steady-state solution and the derivatives of the oscillatoradmittance function in coupled conditions. These derivatives are then introduced into (24)-(26).

Since the dimension of system (25) is $2 N$, where $N$ is the number of oscillator elements, there will be $2 N$ poles. One of them is $\gamma_{1}=0$, due to the autonomy of the global system. This is consistent with the fact that matrix $\left[M_{2}\right]$ is a singular, since the column containing the derivatives with respect to a given phase can be expressed in terms of the rest of phase-derivative columns. The remaining $2 N-1$ poles determine the stability of each steady-state solution. For stability, the $2 N-1$ poles must be on the left-hand side (LHS) of the complex plane.

The stability formulation (24)-(25) has been applied to the in-phase solution of a fully connected topology with $N=5$ and antenna gain $G=3 \mathrm{~dB}$. In Fig. 13, the real part of the poles has been traced versus $d$. To get insight into the behavior, two different distance intervals have been considered: a longer one, going from $7 \mathrm{~m}$ to $8 \mathrm{~m}$ [Fig. 13(a)] and a shorter one, going from $1.1 \mathrm{~m}$ to $1.4 \mathrm{~m}$ [Fig. 13(b)]. The long interval shows a quasi-periodicity of the stable sections versus the distance $d$. The stability margin is smaller at larger distance values [compare (a) with (b)], due to the weaker coupling conditions. The shorter interval evidences two distinct instability mechanisms. The first one is associated with turning points, at which a real pole crosses the imaginary axis. The pole at zero gives rise to an infinite slope of the curve, which leads to a coexistence of steady-state solutions. The real pole passes through zero with an infinite slope versus $d$. The second mechanism is the Hopf bifurcation, at which a pair of complex-conjugate poles crosses the imaginary axis.

The circuit-level stability analysis of the multi-oscillator configuration cannot be based on a reduced circuit with only one or two oscillators (in the star case), since the small perturbations will be different for all the oscillator elements. The results of Fig. 13 could not be compared with pole-zero identification [38]-[39] due to the impossibility to account for the frequency dependence of the coupling networks in the conversion-matrix approach, as implemented on commercial HB. HB convergence problems were also experience in the multi-oscillator system. Instead, the predictions of the semianalytical method have been validated with measurements, in the case of the interval $1.1 \mathrm{~m}$ to $1.4 \mathrm{~m}$. The other interval was discarded due to the limited size of the laboratory. The measured stable sections are indicated with arrows in Fig. 13(b).

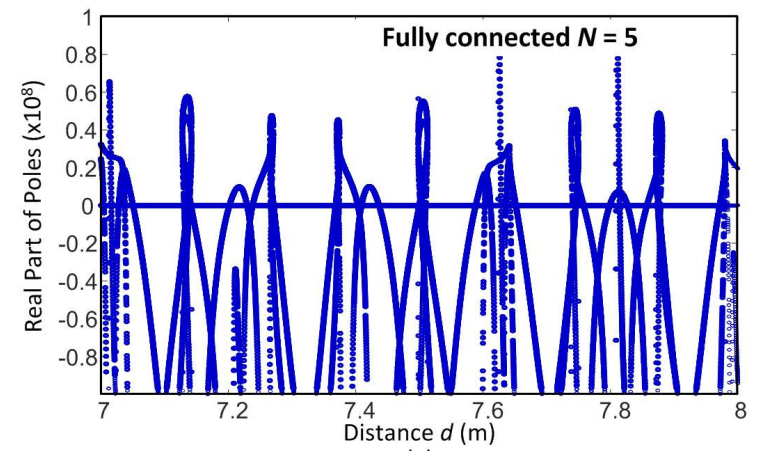

(a)

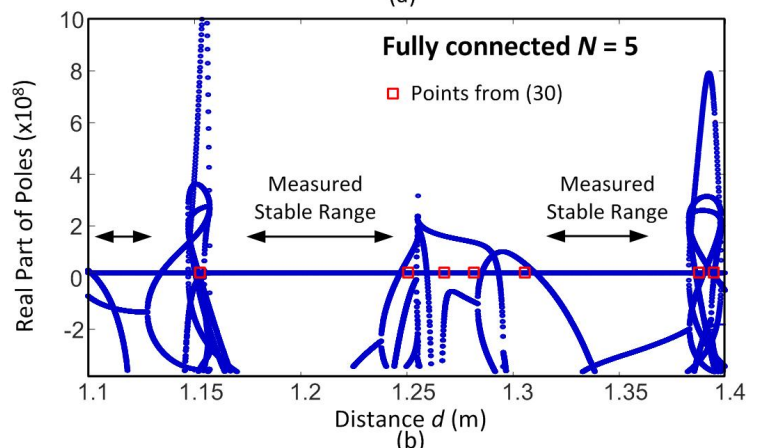

Fig. 13 Variation of the real part of the poles versus $d$. Two different distance intervals have been considered: (a) a longer one, from $7 \mathrm{~m}$ to $8 \mathrm{~m}$ and (b) a shorter one, from $1.1 \mathrm{~m}$ to $1.4 \mathrm{~m}$.

Fig. 14 presents the stability analysis of the in-phase mode of the fully connected topology for different numbers of oscillator elements, from $N=4$ to $N=6$. In the three cases, the real part of the poles has been traced versus the distance $d$. The antenna gain has a significant impact on the stability properties. For too low gain, the stability margins will be small, due to the weak coupling conditions. For too high gain, the system may become unstable, due to a high sensitivity to perturbations, which will be different in the various oscillator elements. In general terms, to ensure stable distance intervals, gain must be reduced when increasing $N$. Comparing Fig. 14(a) and Fig. 14(b) presenting the stability results for $N=4$ and $N=5$, respectively, and the same antenna gain $G=3 \mathrm{~dB}$, less stable sections are obtained for $N=4$. In order to have similar stable intervals, the antenna gain for $N=6$ has been reduced to $G=0 \mathrm{~dB}$. The measured stable interval is indicated with arrows in the cases $N=4$ and $N=5$. A more detailed comparison between the simulation predictions and the experimental results is shown in Table III. 
TABLE III.

STABLE DISTANCE RANGE FOR THE IN-PHASE MODE OF THE FULLYCONNECTED TOPOLOGY.

\begin{tabular}{|c||c||c||c|}
\hline $\begin{array}{c}\boldsymbol{N} \\
\text { oscillators }\end{array}$ & $\begin{array}{c}\text { Stable Simulated } \\
\text { Range (m) }\end{array}$ & $\begin{array}{c}\text { Total } \\
\text { Length } \\
\mathbf{( c m )}\end{array}$ & $\begin{array}{c}\text { Measured } \\
\text { Range } \\
(\mathbf{m})\end{array}$ \\
\hline \hline \multirow{2}{*}{$\mathbf{4}$} & $(1.1,1.136)$ & 3.6 & $(1.1,1.12)$ \\
& $(1.155,1.252)$ & 9.7 & $(1.17,1.25)$ \\
$(G=3 \mathrm{~dB})$ & $(1.287,1.307)$ & 2.0 & 1.3 \\
& $(1.356,1.374)$ & 1.8 & 1.37 \\
\hline $\mathbf{5}$ & $(1.1,1.134)$ & 3.4 & 1.12 \\
& $(1.158,1.248)$ & 9.0 & $(1.18,1.24)$ \\
$(G=3 \mathrm{~dB})$ & $(1.31,1.383)$ & 7.3 & $(1.32,1.36)$ \\
\hline $\mathbf{6}$ & $(1.1,1.118)$ & 1.8 & \\
& $(1.177,1.226)$ & 4.9 & --------- \\
$(G=0 \mathrm{~dB})$ & $(1.309,1.361)$ & 5.2 & \\
\hline \multicolumn{3}{|c|}{} \\
\end{tabular}
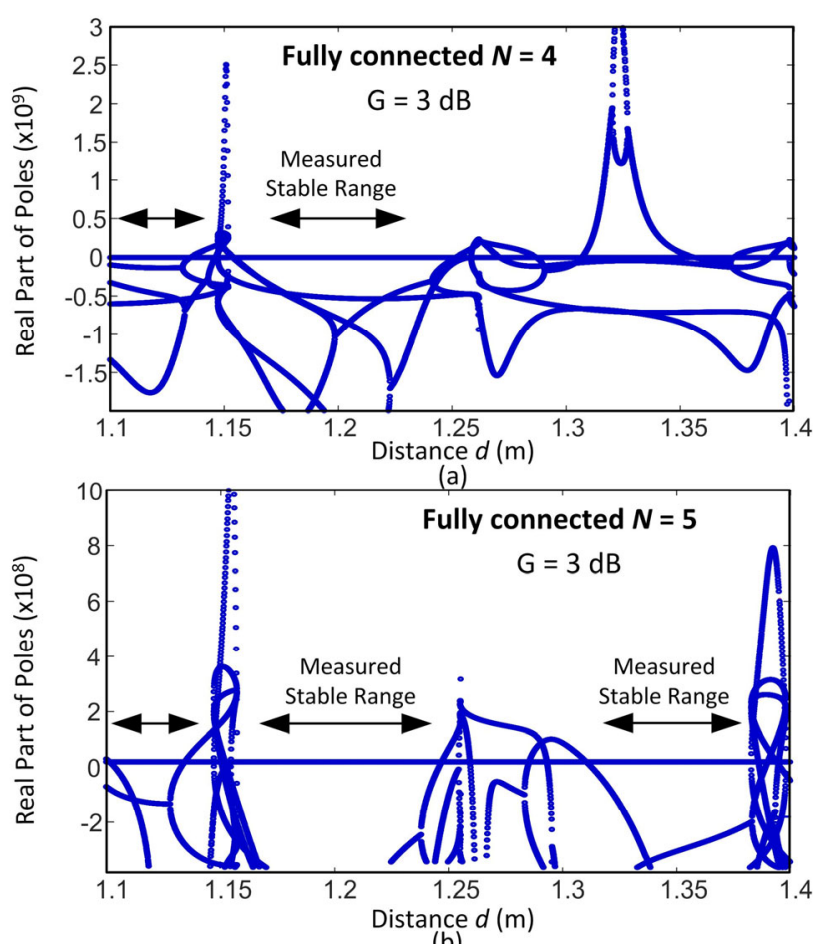

(b)

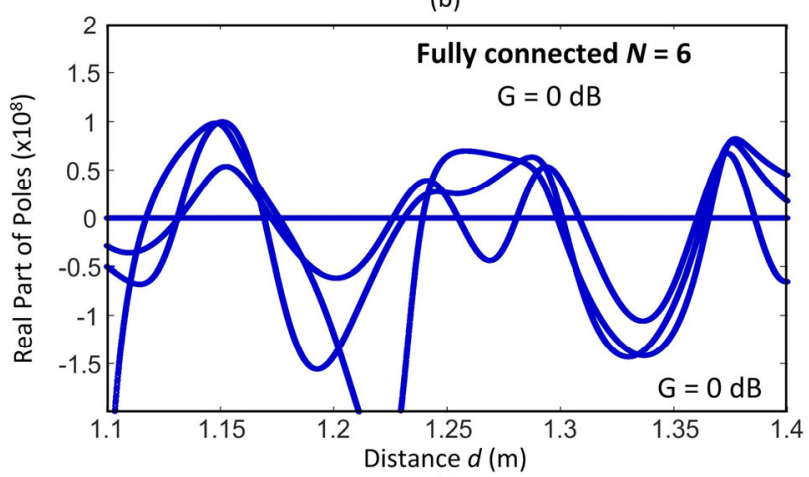

(c)

Fig. 14 Stability analysis of the in-phase mode of the fully connected topology for different number of oscillator elements. In all cases, the real part of the poles obtained with (24) has been traced versus the distance $d$. The measured stable interval is indicated with arrows in the three figures. (a) $N=4$. (b) $N=5$. (c) $N=6$.

When varying with $d$, the matrixes $[C]$ and $[\partial C / \partial(j \omega)]$ exhibit significant changes, affecting the stability properties. Due to the high order of the matrixes in (24) and the need to split these matrixes into real and imaginary parts, it is difficult to provide an intuitive explanation of the stability changes. However, from an inspection of the pole patterns, under low antenna gain, Hopf bifurcations usually take place near the minimum and maximum frequency excursions versus $d$. The observation is consistent with the general behavior of injection-locked oscillators under a small input signal, where qualitative stability changes are also observed at the frequency extremes. In this particular case, the stable sections exhibit a negative frequency slope versus $d$. To provide a hint on the distribution of the stable and unstable intervals, the frequency extremes have been calculated with the following general steady-state equation, valid for all the symmetric topologies:

$$
Y_{v} \Delta V+Y_{\omega} \Delta \omega=\lambda\left(n, N, \omega_{s}, d\right)
$$

If the distance undergoes a small increment $\Delta d$, the increments undergone by $\Delta V, \Delta \omega_{s}$ at the next point of the solution curve can be estimated through the following linearization:

$$
\begin{aligned}
& G_{v} \Delta V+\left[G_{\omega}-\lambda_{\omega}{ }^{r}\left(n, N, \omega_{s}, d\right)\right] \Delta \omega_{s}=\lambda_{d}{ }^{r}\left(n, N, \omega_{s}, d\right) \Delta d \\
& B_{v} \Delta V+\left[B_{\omega}-\lambda_{\omega}{ }^{i}\left(n, N, \omega_{s}, d\right)\right] \Delta \omega_{s}=\lambda_{d}{ }^{i}\left(n, N, \omega_{s}, d\right) \Delta d
\end{aligned}
$$

where the subindex indicates the variable with respect to which $\lambda$ is differentiated and the superindex indicates real and imaginary parts. Solving for $\Delta d$ in terms of $\Delta \omega_{s}$ and making the two incremental quantities tend to zero, one easily obtains that the extreme condition $\partial \omega_{s} / \partial d=0$ implies:

$$
G_{v} \lambda_{d}{ }^{i}\left(n, N, \omega_{s}, d\right)-B_{v} \lambda_{d}{ }^{r}\left(n, N, \omega_{s}, d\right)=0
$$

For illustration, the case of a fully connected configuration with $N=5$ will be considered. The derivatives of $\lambda$ are:

$$
\begin{aligned}
& \lambda_{d}^{r}=-A / d\left[\beta(n) \cos \left(\omega_{s} d / c\right)+\gamma(n) / \delta \cos \left(\omega_{s} d \delta / c\right)\right]- \\
& -A \omega_{s} / c\left[\beta(n) \sin \left(\omega_{s} d / c\right)+\gamma(n) \sin \left(\omega_{s} d \delta / c\right)\right] \\
& \lambda_{d}^{i}=A / d\left[\beta(n) \sin \left(\omega_{s} d / c\right)+\gamma(n) / \delta \sin \left(\omega_{s} d \delta / c\right)\right]- \\
& -A \omega_{s} / c\left[\beta(n) \cos \left(\omega_{s} d / c\right)+\gamma(n) \cos \left(\omega_{s} d \delta / c\right)\right]
\end{aligned}
$$

Expressions (30) and (31) are in agreement with the quasiperiodicity observed in the stable sections of Fig. 13(a). The points obtained through (30) have been superimposed with squares in Fig. 13(b). From (30)-(31), different intervals of multivalued solutions can be expected for the distinct modes, due to the different values of $\beta(n), \gamma(n)$. Nevertheless, one should emphasize that this study provides just an approximation, enabling some analytical insight.

As easily derived from (29), turning points versus $d$ (satisfying $\partial \omega_{s} / \partial d \rightarrow \infty$ ) will occur at the $d$ values at which the following condition is fulfilled:

$$
G_{v}\left[B_{\omega}+\lambda_{\omega}^{i}\left(n, N, \omega_{s}, d\right)\right]-B_{v}\left[G_{\omega}+\lambda_{\omega}^{r}\left(n, N, \omega_{s}, d\right)\right]=0
$$

which depends on the frequency derivative of $\lambda$, given by: 


$$
\lambda_{\omega}=-A\left(1 / \omega_{s}+j d / c\right)\left[\beta(n) e^{-j \omega_{s} d / c}+\gamma(n) e^{-j \omega_{s} d \delta / c}\right]
$$

Since $G_{v} B_{\omega}-B_{v} G_{\omega}>0$ in a stable free-running oscillator [37], [40]-[41], condition (32) can only be fulfilled for sufficiently large magnitude $A$ of the equivalent coupling admittance $\lambda$. This explains why turning points are only observed from a sufficiently high antenna gain. As gathered from (32)-(33), turning points also exhibit a quasi-periodic pattern versus $d$. Their location of the turning points will also be different for the various oscillation modes. When a mode becomes unstable, the system is likely evolve to a different oscillation mode with different phase shifts. Fig. 15 presents the stability analysis of the $144^{\circ}$ and $72^{\circ}$ modes in the fully connected topology with $N=5$ and $G=3 \mathrm{~dB}$. The stable sections of the thwo modes are smaller than those corresponding to the $0^{\circ}$ mode. For the $d$ values where two or more modes are stable, the physical observation of any of these modes is possible, depending on the initial conditions. Thus, operation in the multi-valued regions should be avoided.

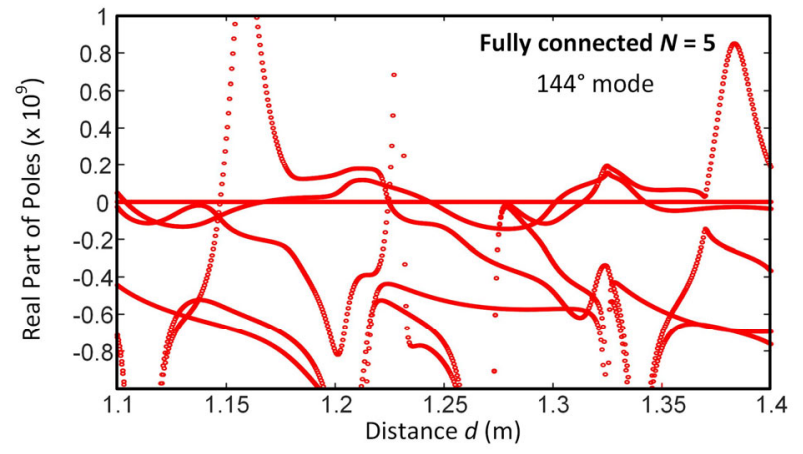

(a)

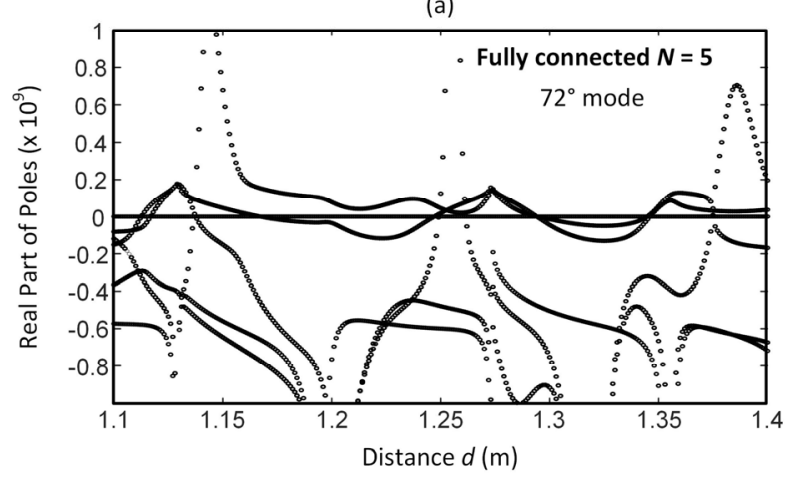

(b)

Fig. 15 Stability analysis of the two other modes existing in the fullyconnected topology with $N=5$ and $G=3 \mathrm{~dB}$. The real part of the poles has been represented versus the distance $d$. (a) $144^{\circ}$ mode. (b) $72^{\circ}$ mode.

\section{DisCREPANCIES BETWEEN THE OSCILLATOR ELEMENTS}

For equal oscillator elements, in a given oscillation mode, the system exhibits equal node amplitude $V_{s}$ and a certain constant phase shift $\phi_{s}$ between the elements. However, in the presence of the discrepant oscillators, the node amplitudes will no longer be equal and the oscillator phases will be different from those corresponding to the modes of the symmetric system in (8). Nevertheless, if the increments $\delta Y_{m, v}, \delta Y_{m, \omega}$ with respect to the ideally identical values are small, it will be possible to obtain the new phase and amplitude distributions from a first-order Taylor series expansion of the system equations, about the symmetric steady-state mode. The Taylor series expansion is:

$$
\begin{aligned}
& \left\{\left[\frac{\partial Y\left(\bar{V}_{s}, \omega_{s}\right)}{\partial \bar{V}}\right]\left[V_{s, m} e^{j \phi_{s, m}}\right]_{d}\right\} \delta \bar{V}_{s}+ \\
& \left\{\left[Y\left(\bar{V}_{s}, \omega_{s}\right)\right]-[C]\right\}\left[j V_{s, m} e^{j \phi_{s, m}}\right]_{d} \delta \bar{\phi}_{s}+ \\
& \left\{\left[\frac{\partial Y\left(\bar{V}_{s}, \omega_{s}\right)}{\partial \omega}\right]-\left[\frac{\partial C\left(\omega_{s}\right)}{\partial \omega}\right]\right\}\left[V_{s, m} e^{j \phi_{s, m}}\right]_{V} \delta \omega_{s}=\left[V_{s, m} e^{j \phi_{s, m}}\right]_{d} \delta \bar{Y}
\end{aligned}
$$

where the vector $\delta \bar{Y}$ accounts for the increments in the oscillator-admittance functions with respect to those corresponding to the ideal mode. Note that the system has a phase invariance, so the phase origin will be arbitrarily taken at the first oscillator element $\delta \phi_{1}=0$. Thus, the above system is well conditioned, with $2 N$ real equations in $2 N$ unknowns, including $\delta \omega_{s}$. Clearly, for a small increment of these admittance functions, the phase shift distribution and synchronized oscillation frequency should be close to those corresponding to the original mode, as the system approaches a homogeneous case, with zero increments. In general terms, smaller deviations will be obtained with respect to the ideal symmetric mode for higher magnitudes of the various derivatives of the left-hand side of (34). Fig. 16 presents a quantitative analysis of the deviations in the oscillation frequency and the phase of one of the oscillator elements in a fully-connected system with $N=5$. Just for illustration, random increments of various magnitude orders, between $10^{-5}$ $\Omega^{-1}$ and $10^{-2} \Omega^{-1}$ (beyond the range of validity of the linearization), have been considered in the real and imaginary parts of the oscillator admittances (with respect to the ideal identical values). As can be seen, the phase errors remain small provided that the increments are moderate. The discrepancies can be compensated by tuning the oscillator elements.
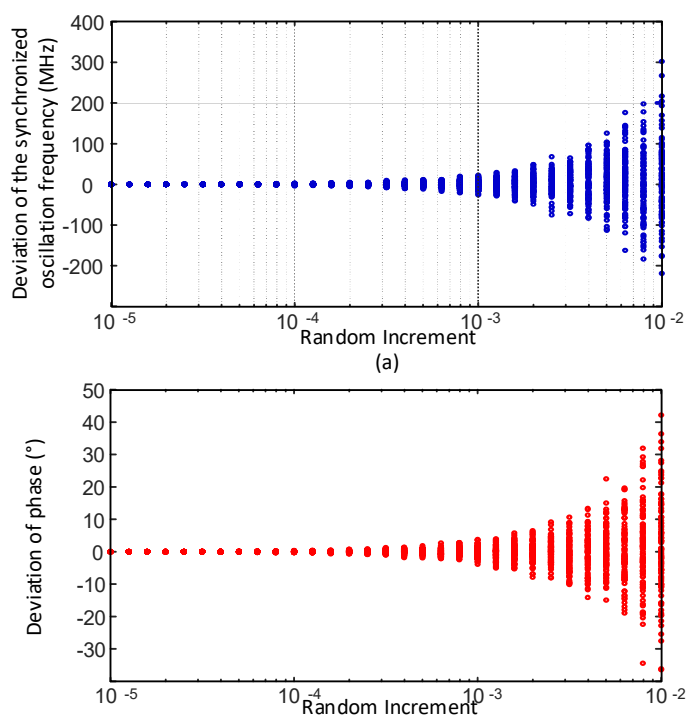

(b)

Fig. 16 Quantitative analysis of the deviations in the oscillation frequency and the phase of one of the oscillator elements in a fully-connected system with 
$N=5$. Random increments of various magnitude orders, between $10^{-5} \Omega^{-1}$ and $10^{-2} \Omega^{-1}$, have been considered in the real and imaginary parts of the oscillator admittances (with respect to the ideal identical values).

\section{PhASE-NOISE ANALYSIS}

For the phase-noise analysis, an equivalent noise current source is introduced in parallel at the node where each oscillator is connected to the coupled network. These noise sources are calculated so as to match the oscillator phase-noise spectrum in free-running conditions, as described in [41]. In the presence of the equivalent noise sources, the perturbed system is:

$$
\begin{aligned}
& \left\{\left[\frac{\partial Y\left(\bar{V}_{s}, \omega_{s}\right)}{\partial \bar{V}}\right]\left[V_{s, m} e^{j \phi_{s, m}}\right]_{d}\right\} \delta \bar{V}_{s}(t)+ \\
& \left\{\left[Y\left(\bar{V}_{s}, \omega_{s}\right)\right]-[C]\right\}\left[j V_{s, m} e^{j \phi_{s, m}}\right]_{d} \delta \bar{\phi}_{s}(t)+ \\
& \left\{\left[\frac{\partial Y\left(\bar{V}_{s}, \omega_{s}\right)}{\partial j \omega}\right]-\left[\frac{\partial C\left(\omega_{s}\right)}{\partial j \omega}\right]\right\}\left[V_{s, m} e^{j \phi_{s, m}}\right]_{d}\left[\frac{1}{V_{s, m}} \delta \dot{V}_{s, m}(t)+j \delta \dot{\phi}_{s, m}(t)\right]_{V}= \\
& \bar{I}_{N}(t)
\end{aligned}
$$

where $\bar{I}_{N}(t)$ is the vector containing the time-varying noise sources. Consideration of flicker noise would require an additional sub-system, relating the baseband components of the voltages and phases to the low-frequency sources [33]. Unlike the case of perturbations about the fundamental frequency, the baseband ones can be assumed as uncoupled, as they are internal to the oscillator elements. Another possibility is to include the flicker-noise contribution into the vector $N$ by considering an additional term, involving the derivative of the admittance function of each oscillator with respect to a DC current source, in a manner similar to what is done in [40], [43]. Such a derivative is also calculated through finite differences. This approach has been followed here to account for the flicker noise, included in the equivalent noise sources $\bar{I}_{N}(t)$.

To obtain the phase noise spectrum of each oscillator in the coupled system, equation (35) is split into real and imaginary parts. Applying the Fourier transform, one obtains the following compact system [21]-[22]:

$$
\left\{\left[M_{1}\right] j \Omega-\left[M_{2}\right]\right\}\left[\begin{array}{c}
\delta \bar{V}_{s}(\Omega) \\
\delta \bar{\phi}_{s}(\Omega)
\end{array}\right]=\frac{\bar{I}_{N}(\Omega)}{V}
$$

where $\Omega$ is the offset frequency from the carrier and equal oscillation amplitude $V$ (as in all the symmetric configurations) have been considered. The matrixes $\left[M_{1}\right]$ and $\left[M_{2}\right]$ are the same ones appearing in (25). To obtain the phase noise spectrum $\left|\delta \phi_{s, m}(\Omega)\right|^{2}$ one should take into account that all the distinct noise sources as well as the real and imaginary parts of each noise are uncorrelated [35], [37], [44]. From inspection of system (35), in a symmetric configuration with the same steady-state amplitudes and phases at all the oscillator elements, the amplitude and phase-noise spectra will be identical at all the oscillators. As already indicated, the matrix $\left[M_{2}\right]$ is singular. Thus, at low offset frequencies one can expect a decay of $-30 \mathrm{~dB}$ per decade when flicker noise is considered, in consistency with the free running operation of the whole system.

The phase-noise analysis described has been applied to the in-phase solution of the fully connected system of $N=5$ elements. The results corresponding to the distance $d=1.2 \mathrm{~m}$ and $d=1.35 \mathrm{~m}$ are presented in Fig. 17(a). The simulated phase-noise spectra of all the oscillator elements are superimposed. However, the measured spectra exhibit small discrepancies, due to tolerances. The phase noise is higher for $d=1.35 \mathrm{~m}$ than for $d=1.2 \mathrm{~m}$, which is attributed to the smaller stability margin for $d=1.35 \mathrm{~m}$ [see Fig. 14(b)].

The phase noise has been compared with that of a single free-running oscillator, isolated from the rest, also represented in Fig. 17(a). The phase-noise of the isolated oscillator is higher than that of the oscillators in the synchronized coupled system. In the standalone free-running oscillator there is more disagreement between simulation and measurements, attributed to the fact that in this higher noise case our measurement was not very accurate.

Obtaining a closed-form expression of the phase-noise spectrum in terms of the coupling coefficients and other system parameters is virtually impossible. However, in order to give a hint on the coupling effects we have considered a system with two oscillators only. Neglecting the amplitude perturbations, in the presence of a real coupling coefficient $C_{r}$, the phase noise spectrum of the in-phase solution is:

$$
|\delta \phi(\Omega)|^{2}=\frac{\left(2 C_{r}^{2}+B_{\omega}^{2} \Omega^{2}\right) 2\left|I_{N}\right|^{2}}{\Omega^{2}\left(B_{\omega}^{4} \Omega^{2}+4 C_{r}^{2} B_{\omega}^{2}\right) V^{2}}
$$

where the frequency derivative of the coupling coefficient has also been neglected for better insight. Near the carrier frequency, the phase noise reduction with respect to the freerunning case (corresponding to $C_{r}=0$ ) is $10 \log (2)$. A higher reduction can be expected for a higher number of oscillator elements, in agreement with [44]. The fully-connected system with $N=5$ analyzed here has two different coupling coefficients [see (14)], with a significant frequency variation that will also affect the noise spectrum, as gathered from (35).

The spectrum will become noisier when operating near the stability boundaries. This is shown in Fig. 17(b), which presents the variation of the phase-noise spectral density at the constant offset frequency $100 \mathrm{kHz}$. Predictions by the perturbation system (35) are compared with the experimental measurement of the phase-noise spectral density and exhibit a reasonable agreement. The analysis in Fig. 17(b) provides a good insight into the effect of a low stability margin. One must take into account that the determinant of the matrix on the left side of (36) agrees with the characteristic determinant of the stability formulation in (25) when evaluated at $j \Omega$ instead of the complex frequency $s$. Near a bifurcation point, the common denominator of the amplitude and phase perturbations approaches a singularity condition at an offset frequency agreeing with the frequency of the critical poles. In the case of the system considered in this work, the frequencies of the critical poles are slightly higher than $100 \mathrm{MHz}$. Therefore, in the phase-noise analysis interval the bump is not observable. However, there is an increase of the phase-noise spectral density, affected by the presence of the bump. Near 
the turning points, which implies an additional singularity at zero frequency offset, there is an increase of the phase-noise spectral density at low offset frequencies.

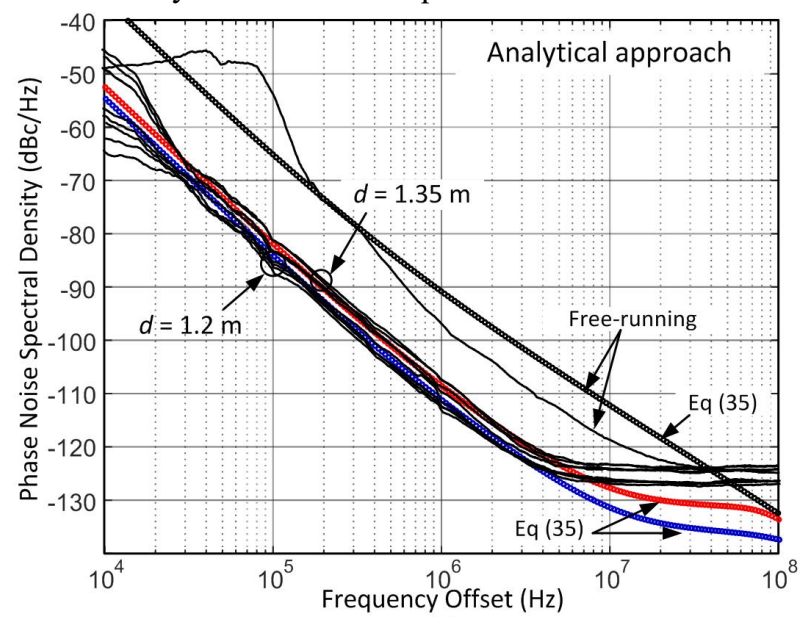

(a)

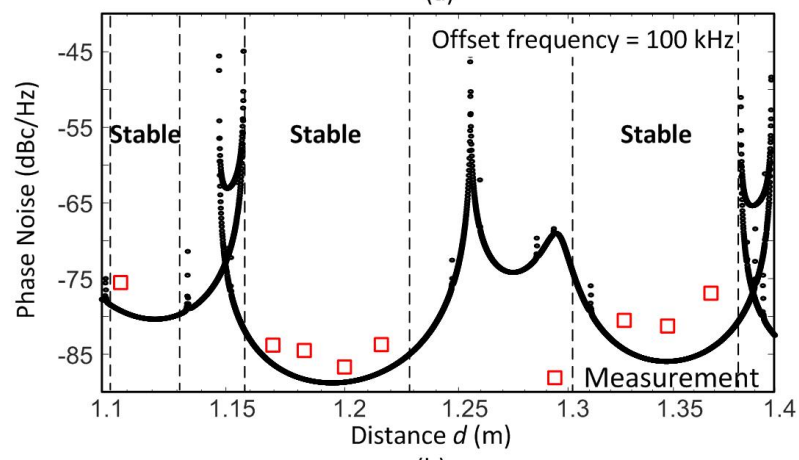

(b)

Fig. 17 Phase-noise analysis of a fully-connected system of $N=5$ elements. (a) Predictions by the perturbation system (35) for two distance values $d=1.2 \mathrm{~m}$ and $d=1.35 \mathrm{~m}$ are compared with the experimental measurement of the phase-noise spectral density. (b) Variation of the phase-noise spectral density with the distance $d$ at the constant offset frequency $f_{\text {off }}=100 \mathrm{kHz}$

\section{COnClusion}

An in-depth study of wireless-coupled oscillator networks for clock-signal synchronization has been presented. The investigation covers three main configurations, with ring, fully connected and star topologies. In each case, an equivalent coupling-admittance matrix has been derived, obtaining the possible oscillation modes through an eigenvalue/eigenvector calculation. A methodology for an efficient harmonic-balance simulation of the system, using a reduced-equivalent circuit, has been presented. This simulation is complemented with an approximate semi-analytical methodology, which is able to provide the complete solution curves obtained for the different oscillation modes, often comprising multi-valued sections of high complexity. The stability properties and phase-noise behavior are evaluated with a perturbation formulation, relying on derivatives of the individual oscillator admittance function, calculated through finite differences in harmonic balance. The effect of oscillator tolerances is predicted through a linearization of the coupled system about each particular mode. All the new formulations and reduced HB analyses have been validated through costly circuit-level simulations of the entire coupled system and with measurements.

\section{REFERENCES}

[1] O. Simeone, U. Spagnolini, Y. Bar-Ness, and S. H. Strogatz, "Distributed synchronization in wireless networks," IEEE Signal Process. Mag., vol. 25, no. 5, pp. 81-97, Sep. 2008.

[2] D. J. Jörg et al., "Synchronization of mutually coupled digital PLLs in massive MIMO systems," IEEE Int. Conf. Commun., London, UK, 2015, pp. 1716-1721.

[3] O. Simenone and U. Spagnolini, "Distributed synchronization for wireless sensor networks with coupled discrete-time oscillators, Eurasip J. Wireless Commun. Networking, \#57054, 2007.

[4] S. Barbarossa and G. Scutari, "Decentralized maximum likelihood estimation for sensor networks composed of nonlinearly coupled dynamical systems," IEEE Trans. Signal Process., vol. 55, no. 7, pp. 3456-3470, Jul. 2007.

[5] A. Mauroy, P. Sacré, and R. Sepulchre, "Kick synchronization versus diffusive synchronization," 51st IEEE Conf. Decision and Control (CDC), Maui, HI, 2012, pp. 7171-7183.

[6] K. Matsuzaka, K. Nakada, and T. Morie, "Analog CMOS circuit implementation of a system of pulse-coupled oscillators for spike-based computation," IEEE Int. Symp. Circuits Syst. (ISCAS), Rio de Janeiro, 2011, pp. 2849-2852.

[7] E. Mallada, R. A. Freeman, and A. K. Tang, "Distributed Synchronization of Heterogeneous Oscillators on Networks With Arbitrary Topology," IEEE Trans. Control Netw. Syst., vol. 3, no. 1, pp. 12-23, Mar. 2016.

[8] Y. Wang, F. Nunez and F. J. Doyle, "Energy-Efficient Pulse-Coupled Synchronization Strategy Design for Wireless Sensor Networks Through Reduced Idle Listening," IEEE Trans. Signal Proces., vol. 60, no. 10, pp. 5293-5306, Oct. 2012.

[9] O. Simeone and G. Scutari, "Pulse-coupled distributed PLLs in heterogeneous wireless networks," 2007 Conference Record of the Forty-First Asilomar Conference on Signals, Systems and Computers, Pacific Grove, CA, 2007, pp. 1770-1775.

[10] C. H. Rentel and T. Kunz, "A Mutual Network Synchronization Method for Wireless Ad Hoc and Sensor Networks," IEEE Trans. Mobile Comput., vol. 7, no. 5, pp. 633-646, May 2008.

[11] E. Mallada, X. Meng, M. Hack, L. Zhang and A. Tang, "Skewless Network Clock Synchronization Without Discontinuity: Convergence and Performance," in IEEE/ACM Trans. Netw., vol. 23, no. 5, pp. 16191633, Oct. 2015.

[12] A. Gushchin, E. Mallada and A. Tang, "Phase-Coupled Oscillators with Plastic Coupling: Synchronization and Stability," IEEE Trans. Netw. Sci. Eng., vol. 3, no. 4, pp. 240-256, Oct.-Dec. 12016.

[13] A. Díaz-Guilera, A. Arenas, Phase patterns of coupled oscillators with application to wireless communication, P. Lio, et al. (Eds.), BIOWIRE 2007, LNCS, vol. 5151, Springer-Verlag, Berlin (2008), pp. 172-179.

[14] M. Pontón and A. Suárez, "Stability analysis of wireless coupledoscillator circuits," IEEE MTT-S Int. Microwave Symp., Honolulu, Hawaii, Jun. 2017.

[15] R. A. York, "Nonlinear analysis of phase relationships in quasi-optical oscillator arrays," IEEE Trans. Microw. Theory Techn., vol. 41, pp. 1799-1809, Oct. 1993.

[16] R. A. York and T. Itoh, "Injection- and phase-locking techniques for beam control," IEEE Trans. Microw. Theory Techn., vol. 46, pp. 19201929, 1998.

[17] T. Heath, R. R. Kerr, and G. D. Hopkins, Two-dimensional, nonlinear oscillator array antenna, IEEE Aerosp. Conf., pp. 1104-1115, 2005.

[18] T. Heath, R. R. Kerr, and G. D. Hopkins, "Nonlinear oscillator array antenna development at GTRI,” IEEE Aerosp. Conf., p. 19, 2006.

[19] R. J. Pogorzelski, P. F. Maccarini and R. A. York, "A continuum model of the dynamics of coupled oscillator arrays for phase-shifterless beam scanning," IEEE Trans. Microw. Theory Techn., vol. 47, no. 4, pp. 463470, Apr. 1999. 
[20] R. J. Pogorzelski, "On the steady-state phase distribution in a hexagonally coupled oscillator array," IEEE Trans. Antennas Propag., vol. 53, no. 12, pp. 4058-4064, Dec. 2005.

[21] A. Georgiadis, A. Collado and A. Suárez, "New techniques for the analysis and design of coupled-oscillator systems," IEEE Trans. Microw. Theory Techn, vol. 54, pp. 3864-3877, Nov., 2006.

[22] A. Suárez, F. Ramírez and S. Sancho, "Stability and Noise Analysis of Coupled-Oscillator Systems," IEEE Trans. Microw. Theory Techn., vol. 59, no. 4, pp. 1032-1046, Apr. 2011.

[23] A. Suárez, F. Ramírez, S. Sancho and J.M. Collantes, "Global stability analysis of coupled-oscillator systems," IEEE Trans. Microw. Theory Techn., vol. 63, no. 1, pp. 165 - 180, Jan. 2015.

[24] A. Suárez, S. Sancho and F. Ramírez, "General Formulation for the Analysis of Injection-Locked Coupled-Oscillator Systems," IEEE Trans. Microw. Theory Techn., vol. 61, no. 12, pp. 4730-4744, Dec., 2013.

[25] A. Collado, F. Ramírez, A. Suárez and J. P. Pascual, "Harmonic-balance analysis and synthesis of coupled-oscillator arrays," IEEE Microw. and Wireless Comp. Lett., vol. 14, pp. 192, May, 2004.

[26] A. Suárez, A. Collado and F. Ramírez, "Harmonic-balance techniques for the design of coupled-oscillator systems in both unforced and injection-locked operation," IEEE MTT-S Int. Microwave Symp. Dig., Long Beach, CA, USA, Jun., 2005.

[27] Y. M. Tousi, V. Pourahmad and E. Afshari "Frequency tuning of terahertz sources using delay-coupled oscillators" Phys. Rev. Lett., vol. 108 pp. 234101.1-234101.5 Jun. 2012.

[28] M. Adnan and E. Afshari, "A 105-GHz VCO with 9.5\% Tuning Range and 2.8-mW Peak Output Power in a 65-nm Bulk CMOS Process," IEEE Trans. Microw. Theory Techn, vol. 62, no. 4, pp. 753-762, April 2014.

[29] K. Kurokawa, "An Analysis of Rucker's Multidevice Symmetrical Oscillator," IEEE Trans. Microw. Theory Techn., vol. 18, pp. 967, May. 1970.

[30] C. Balanis, Antenna Theory. Analysis and Design. John Wiley \& Sons, 2005.

[31] M. Pontón and A. Suárez, "Wireless Injection Locking of Oscillator Circuits," IEEE Trans. Microw. Theory Techn., vol. 64, no. 12, pp. 4646-4659, Dec. 2016.

[32] F. Ramírez, E. de Cos and A. Suárez, "Nonlinear analysis tools for the optimized design of harmonic-injection frequency dividers", IEEE Trans. Microw. Theory Techn., vol. 51, no. 6, pp. 1752-1762, Jun. 2003.

[33] A. Suárez, Analysis and Design of Autonomous Microwave Circuits. Hoboken, NJ: Wiley IEEE Pres, 2009.

[34] A. Suárez, R. Quéré, Stability Analysis of Nonlinear Microwave Circuits, Artech-House Publishers, Boston, 2003.

[35] R. Quere, E. Ngoya, M. Camiade, A. Suarez, M. Hessane, and J. Obregon, "Large signal design of broadband monolithic microwave frequency dividers and phase-locked oscillators," IEEE Trans. Microw. Theory Techn., vol. 41, no. 11, pp. 1928-1938, Nov. 1993.

[36] F. Núñez, Y. Wang and F. J. Doyle, "Synchronization of Pulse-Coupled Oscillators on (Strongly) Connected Graphs," IEEE Trans. Autom. Control, vol. 60, no. 6, pp. 1710-1715, Jun. 2015.

[37] K. Kurokawa, "Some basic characteristics of broadband negative resistance oscillators," The Bell System Technical Journal, vol. 48, no. 6, pp. 1937-1955, Jul. -Aug. 1969.

[38] J. Jugo, J. Portilla, A. Anakabe, A. Suárez, and J. M. Collantes, “Closedloop stability analysis of microwave amplifiers," Electron. Lett., vol. 37, no. 4, pp. 226-228, Mar. 2001.

[39] J. M. Collantes, I. Lizarraga, A. Anakabe, and J. Jugo, "Stability verification of microwave circuits through Floquet multiplier analysis", Proc. IEEE Asia-Pacific Circuits Syst., 2004, pp. 997-1000.

[40] K. Kurokawa, "Noise in synchronized oscillators," IEEE Trans. Microw. Theory Techn., vol. 16, no. 4, pp. 234-240, Apr., 1968.

[41] F. Ramírez, M. Pontón, S. Sancho, and A. Suárez, "Phase-Noise Analysis of Injection-Locked Oscillators and Analog Frequency Dividers", IEEE Trans. Microw. Theory Techn., vol. 56, no.2, pp. 393-407, 2008.

[42] V. Rizzoli, F. Mastri, and D. Masotti, "General noise analysis of nonlinear microwave circuits by the piecewise harmonic-balance technique," IEEE Trans. Microw. Theory Techn., vol. 42, no. 5, pp. 807 819, May 1994
[43] M. Pontón and A. Suárez, "Analysis of Two Coupled NLTL-Based Oscillators," IEEE Trans. Microw. Theory Techn., vol. 62, no. 12, pp. 3485-3499, Dec. 2014.

[44] Heng-Chia Chang, Xudong Cao, U.K. Mishra and R.A. York, "Phase noise in coupled oscillators: theory and experiment," IEEE Trans. Microw. Theory Techn., vol. 45, no. 5, pp. 604-615, May 19974.

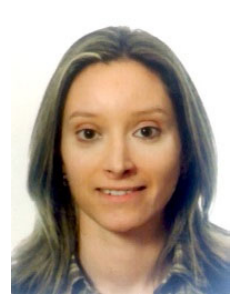

Mabel Pontón (S'08-M'11) was born in Santander, Spain. She received the bachelor's degree in Telecommunication Engineering, master's degree in Information Technologies and Wireless Communications Systems, and Ph.D. degree from the University of Cantabria, Santander, in 2004, 2008, and 2010, respectively. In 2006, she joined the Communications Engineering Department, University of Cantabria.

From 2011 to 2013, she was with the Group of Electronic Design and Applications, Georgia Institute of Technology, Atlanta, GA, USA, as a PostDoctoral Research Fellow.

Her current research interests include the nonlinear analysis and simulation of radiofrequency and microwave circuits, with an emphasis on phase-noise, stability, and bifurcation analysis of complex oscillator topologies.

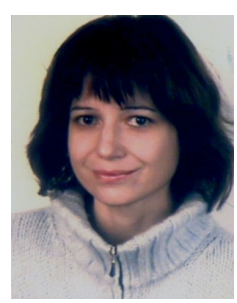

Almudena Suárez (M'96-SM'01-F'12) was born in Santander, Spain. She received the Electronic Physics and Ph.D. degrees from the University of Cantabria, Santander, Spain, in 1987 and 1992, respectively, and the $\mathrm{Ph} . \mathrm{D}$. degree in electronics from the University of Limoges, Limoges, France, in 1993.

She is currently a Full Professor with the Communications Engineering Department, University of Cantabria. She co-authored Stability Analysis of Nonlinear Microwave Circuits (Artech House, 2003) and authored Analysis and Design of Autonomous Microwave Circuits (IEEE-Wiley, 2009).

Prof. Suárez is a member of the Technical Committees of the IEEE International Microwave Symposium (IMS), the European Microwave Conference. She was an IEEE Distinguished Microwave Lecturer from 2006 to 2008, with the talk "Stability analysis and stabilization of power amplifiers." She is the editor-in-chief of International Journal of Microwave and Wireless Technologies, from Cambridge Journals. She is an associate editor of IEEE Microwave Magazine. She is a member of the "Board of Directors" of the European Microwave Association. She was the coordinator of the Communications and Electronic Technology Area for the Spanish National Evaluation and Foresight Agency (ANEP) between 2009 and 2013. 